\title{
Lévy Walk Dynamics in an External Constant Force Field in Non-Static Media
}

\author{
Tian $\mathrm{Zhou}^{1} \cdot$ Pengbo $\mathrm{Xu}^{2} \cdot$ Weihua Deng ${ }^{1}$
}

Received: 17 November 2021 / Accepted: 16 February 2022 / Published online: 28 February 2022

(c) The Author(s), under exclusive licence to Springer Science+Business Media, LLC, part of Springer Nature 2022

\begin{abstract}
Based on the recognition of the huge change of the transport properties for diffusion particles in non-static media, we consider a Lévy walk model subjected to an external constant force in non-static media. Since the physical and comoving coordinates of non-static media are related by scale factor, we equivalently transfer the process from physical coordinate into comoving coordinate and derive the master equation governing the probability density function of the position of the particles in comoving coordinate. Utilizing the Hermite orthogonal polynomial expansions, some statistical properties are obtained, including the asymptotic behaviors of the first two moments in both coordinates and kurtosis. For some representative types of non-static media and Lévy walks, the striking and interesting phenomena originating from the interplay between non-static media, external force, and intrinsic stochastic motion are observed. The stationary distribution are also analyzed for some cases through numerical simulations.
\end{abstract}

Keywords Lévy walk $\cdot$ Non-static media $\cdot$ Constant force $\cdot$ Scale factor $\cdot$ Hermite orthogonal polynomial

\section{Introduction}

The diffusion process attracts the attentions of people since Robert Brown, a Scottish botanist, discovered that pollen and other tiny suspended particles constantly move in an irregular curve

Communicated by Giulio Biroli.

Weihua Deng

dengwh@1zu.edu.cn

Tian Zhou

zhout19@1zu.edu.cn

Pengbo $\mathrm{Xu}$

xupengbo@math.pku.edu.cn

1 Gansu Key Laboratory of Applied Mathematics and Complex Systems, School of Mathematics and Statistics, Lanzhou University, Lanzhou 730000, People's Republic of China

2 School of Mathematical Sciences, Peking University, Beijing 100871, People's Republic of China 
in water in 1827 [4]. For a large group of diffusion processes, their mean square moments (MSDs) behave as $\left\langle x^{2}(t)\right\rangle \sim t^{\alpha}$ after sufficiently long time. The diffusion process is called normal diffusion if $\alpha=1$, otherwise it is termed as anomalous diffusion [5, 42, 57]. A wellknown example of normal diffusion is Brownian motion [48]. One of the famous example for anomalous diffusion is Richardson's research in 1926 [53], in fact it is ubiquitous in a wide range of fields, such as diffusion processes in physics [49], in finance [58], in ecology [50], as well as in biology [30]. Anomalous diffusion can be further subdivided according to the scope of $\alpha$. The further classification of anomalous diffusions leads to subdiffusion for $0<\alpha<1[32,33,59,60,64,67,68]$ and superdiffusion for $\alpha>1[6,12,54]$.

As for the models for diffusion processes, continuous time random walk (CTRW) is one of the central stochastic models based on random walk theory, in which there are two series of independent identical distribution (i.i.d.) random variables; one is waiting time $\tau$ between any two continuous jumps following the distribution $\phi(\tau)$ and another one is jump length $l$ satisfying the probability density function (PDF) $\lambda(l)$ [43-45]. The subdiffusion or superdiffusion process can be viewed as a scaling limit process of CTRW model with infinite $\langle\tau\rangle$ and finite $\left\langle l^{2}\right\rangle$ or finite $\langle\tau\rangle$ and diverging $\left\langle l^{2}\right\rangle$, respectively. If both $\langle\tau\rangle$ and $\left\langle l^{2}\right\rangle$ are finite, it is modeling normal diffusion. A renowned example of superdiffusion is Lévy flight [21, $22,43]$, where the distribution of jump length satisfies $\lambda(l) \sim 1 /|l|^{1+\mu}$ with $0<\mu<2$. For Lévy flight, the transport is usually characterized by its fractional order moments $\left\langle|x|^{\kappa}\right\rangle$ because of the scale-free of jump length $\lambda(l)$. Moreover, [43] shows that $\left\langle|x|^{\kappa}\right\rangle^{2 / \kappa} \simeq t^{2 / \mu}$. Lévy flight is also considered to be an efficient random search model for foraging animals $[51,65,66]$ due to the fractal dimension of their trajectories. Another classical model based on random walk theory to describe anomalous diffusion is Lévy walk. Different from CTRW model, it is spatiotemporally coupled through finite propagation speed $[7,73]$. The traditional one is with constant speed $v_{0}$ and the corresponding running time $\tau$ for each step follows the distribution $\phi(\tau)$. Lévy walk is observed in many fields, such as molecular-motor motion $[13,61]$, human hunter-gatherer foraging [55], optimized robotic search [23], as well as the propagation of COVID-19 pandemic [27].

Almost all the particles move in external potentials [28, 29]. The effects of external forces on Brownian motion are developed by Smoluchowski after Einstein publishing his paper on Brownian motion in 1905. The dynamics of CTRW process under the action of external potentials are discussed in [14, 21, 22, 24, 46] and the authors [34] study Lévy flight process moving in harmonic potential field, in which it is found that the MSD is diversing though a stationary state can be reached. Further, Lévy flights in a harmonic potential well for both overdamped and underdamped situations are detailedly discussed in [15]. Although the generalized Kramers-Fokker-Planck equation of Lévy walk in arbitrary external potentials is given in $[25,26]$, it seems hard to uncover more concrete characters due to the spatiotemporal coupled property. Hermite orthogonal polynomials approximation is a complementary method to integral transform introduced in [69] to solve the spatiotemporal coupled problem of Lévy walk and Lévy walk process under the action of external potential is detailedly discussed in [70, 74] by this brand new method. Moreover, Langevin equations [16] provide a feasible framework to solve the problem with external potentials.

The foundations of the above studies are based on the hypothesis that the medium is static, which means the distance between two unmoved particles does not change with time. In fact, almost all the diffusion processes take place in weakly/strongly non-static media, which can be clearly observed in many fields, such as biology [8, 17, 37, 62], cosmology [1, 9, 10, 35]. Moreover, two typical types of non-staticity of media are expansion and contraction. Diffusion processes taken place in chaotic systems and fluids turn out that the dynamics of the particles alter enormously due to the non-static medium, prompting the related researches $[2,18,19$, 
$31,38,47,63,71,72]$. The corresponding Fokker-Planck equation for random motion in non-static medium is presented in [72] on account of the generalized Chapman-Kolmogorov equation. Besides, the dynamics of CTRW and Lévy walk model in one dimensional nonstatic medium are analyzed in $[38,39,75]$.

It is a widespread phenomenon that the diffusion processes are subjected to an external potential in non-static medium. For example, the particles are usually suffered from interactions in biological media [20, 30, 42] and the diffusion particles may be under a harmonic potential in uniformly expanding medium [40]. Besides, the corresponding Fokker-Planck equation of the CTRW particles moving under the action of an external force in one dimensional uniformly expanding medium is derived in [41]. In this paper, we aim to analyze the transport characters of Lévy walk under the action of external constant force in one dimensional non-static medium.

This paper is organized as follows. In Sect. 2, we introduce the Lévy walk model under the action of an external constant force in one dimensional non-static medium and build its transport equation in the framework of comoving coordinate. In Sect. 3, the corresponding average moments and MSDs in both coordinates are derived through Hermite orthogonal polynomials expansions. We discuss the interplay between a constant force, non-static medium, and the intrinsic stochastic motion in Sect. 4 by considering some representative distributions of running time $\phi(\tau)$ and scale factor $d(t)$ and derive the kurtosis in physical coordinate for the non-static medium with exponential contraction scale factor. Besides, the stationary distribution are also analyzed through numerical simulations for the case of localization in both coordinates. Finally, we conclude the paper with some discussions in Sect. 5.

\section{Lévy Walk Under the Action of an External Constant Force in Non-static Media}

Lévy walk particles with mass $M$ moving in a constant force field $F=M a$, where $a$ represents a constant acceleration, have been considered in one dimensional static medium in [74]. The initial speed of each step is assumed to be $\pm v_{0}$ with equal probability for the direction of left or right. Moreover, $y_{t}$ denotes the location of the particle at time $t$ and $t_{j}$ $(j=1,2,3, \ldots)$ is the time when the $j$ th renewal event was just finished. The running time $\tau:=t_{j}-t_{j-1}$ between two successive renewal events obeys the PDF $\phi(\tau)$. With the above setting, the transport equation between the $j$ th and $(j+1)$ th renewal events satisfies:

$$
\left\{\begin{array}{l}
\frac{d^{2} y_{t_{j}+\tau^{\prime}}}{d \tau^{\prime 2}}=a, \quad \text { for } \tau^{\prime} \in\left(0,\left(t_{j+1} \wedge t\right)-t_{j}\right], \\
\left.\frac{d y_{t_{j}+\tau^{\prime}}}{d \tau^{\prime}}\right|_{\tau^{\prime}=0}= \pm v_{0} .
\end{array}\right.
$$

The solution of Eq. (1) satisfies $y_{t_{j}+\tau^{\prime}}=\frac{1}{2} a \tau^{\prime 2} \pm v_{0} \tau^{\prime}+y_{t_{j}}$ with $\frac{1}{2}$ probability of each them. Comparing with the motion in static medium under the action of an external constant force, the displacement of Lévy walk changes from $\pm v_{0} \tau$ to $\frac{1}{2} a \tau^{2} \pm v_{0} \tau$ when the duration of each step is $\tau$.

In this paper, we focus on analyzing the transport dynamics of Lévy walk under a constant external force field when the one dimensional medium is undergoing uniform expansion or contraction. The relation between the physical coordinate (denoted as $y$ ) and comoving coordinate (denoted as $x$ ) is

$$
y=d(t) x
$$


with $d\left(t_{0}\right)=1$ for the initial time $t_{0}[38,72]$. The function $d(t)$ is named as scale factor from a point of cosmology [52,56]. Furthermore, the medium is expanding if $\dot{d}(t)>0$ and is contracting if $\dot{d}(t)<0$ [40]. It is worth noting that the physical and comoving coordinates are identical at the initial time. The evolution of the physical location $y$ comes from three contributions, which are respectively the intrinsic motion of Lévy walk, the motion induced by constant force, as well as the deterministic motion caused by non-static medium.

The CTRW process in non-static medium is analyzed in [38]. As shown in Fig. 1a, one can assume that the CTRW particle arrives at $y_{n-1}$ at time $t_{n-1}$, then it comes to $y_{n-1}^{\prime}$ at time $t_{n}$ due to the expansion of the medium and further followed by an instantaneous jump with length $\Delta y_{n}$ relative to $y_{n-1}^{\prime}$ because of the intrinsic motion. Since the particle does not move in the comoving coordinate $x$ as long as the walker is in a state of rest, e.g., $x_{n-1}^{+}=x_{n}^{-}$(' ${ }^{+}$' and '-' respectively mean right and left limit), we have the expression of $y_{n-1}^{\prime}$ by combining with (2),

$$
y_{n-1}^{\prime}=\frac{d\left(t_{n}\right)}{d\left(t_{n-1}\right)} y_{n-1} .
$$

The correlation and similarity between CTRW model and the traditional Lévy walk model are stated in $[11,36]$. Because of that, for Lévy walk under the action of constant force in nonstatic medium, as shown in Fig. $1 \mathrm{~b}$, we choose $\xi \in\left(t_{n-1}, t_{n}\right]$, the position $y_{\xi}$ of the Lévy walk particle at time $\xi$ is determined by three parts; one is the deterministic motion caused by nonstatic medium and the corresponding position can be expressed as $y_{\xi}^{\prime}=\frac{d(\xi)}{d\left(t_{n-1}\right)} y_{n-1}$, and the other two are the intrinsic stochastic movement and the motion resulted from constant force, respectively. The displacement induced by the latter two is $\Delta y=\frac{1}{2} a\left(\xi-t_{n-1}\right)^{2} \pm v_{0}\left(\xi-t_{n-1}\right)$
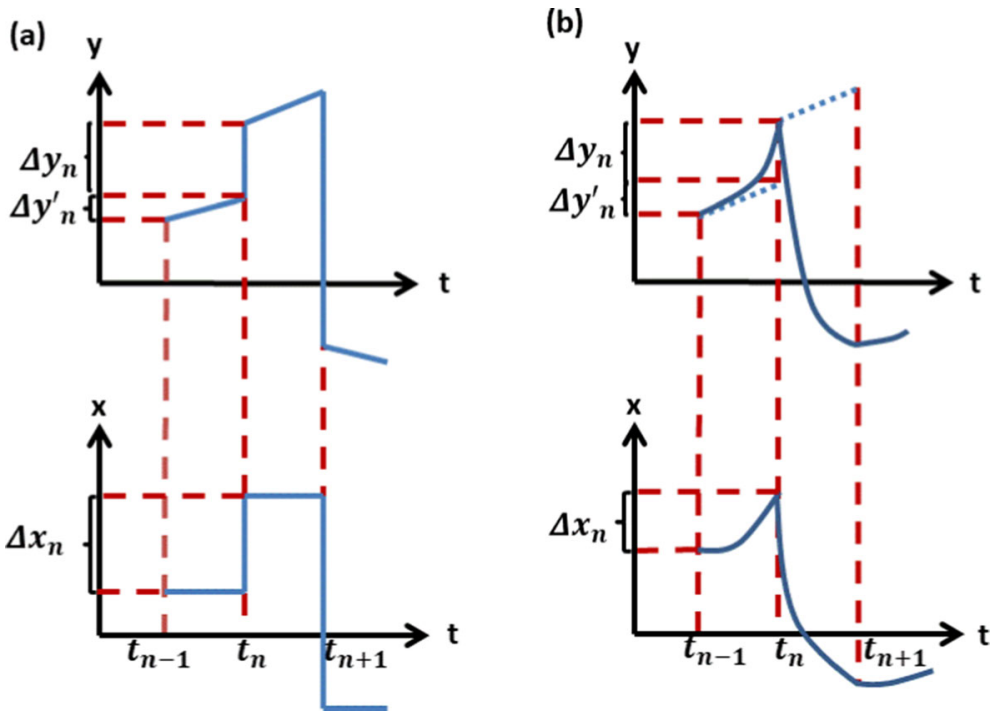

Fig. 1 Legend descriptions of CTRW and Lévy walk under the action of constant force in expanding medium. Here, $x$ and $y$, respectively, denote the comoving and physical coordinates with the relation $y=d(t) x$, where $d(t)$ is the scale factor. $\Delta y_{n}^{\prime}=y_{n-1}^{\prime}-y_{n-1}$ denotes the displacement caused by expanding medium and $\Delta x_{n}=x_{n}-x_{n-1}$ is the displacement $\Delta y_{n}$ in comoving coordinate. a Represents CTRW model in expanding medium and $\Delta y_{n}$ is the jump length drawn from the distribution $\lambda\left(\Delta y_{n}\right)$, and $\mathbf{b}$ is for Lévy walk process under the action of constant force in expanding medium with $\Delta y_{n}=\frac{1}{2} a \tau^{2} \pm v_{0} \tau$, where $\tau$ obeys the distribution $\phi(\tau)$ 
relative to $y_{\xi}^{\prime}$. Then, for the final observation time $t$, the position of the Lévy walk particle is

$$
y_{t}=\frac{d(t)}{d\left(t_{n}\right)} y_{n}+\frac{1}{2} a\left(t-t_{n}\right)^{2} \pm v_{0}\left(t-t_{n}\right) .
$$

Obviously, we recover the results in [74] when the scale factor of the media is $d(t)=1$, which further indicates the distance between two unmoved particles does not change with time.

As discussed above, the final position of the Lévy walk particle in each finished (or unfinished) step in physical coordinate is related to the displacement $\Delta y$ and the location after the deterministic motion induced by non-static medium. In addition to the constant acceleration $a$ and velocity $v_{0}$, the displacement $\Delta y$ is also determined by the running time $\tau$ drawn from $\phi(\tau)$ or the survival time (still denoted as $\tau$ ), which obeys the distribution

$$
\psi(\tau)=\int_{\tau}^{\infty} \phi\left(\tau^{\prime}\right) d \tau^{\prime}
$$

Therefore, the corresponding distribution of $\Delta y$ with running time $\tau$ satisfies the conditional density

$$
\lambda(\triangle y ; \tau)=\frac{1}{2} \delta\left(\Delta y-\frac{1}{2} a \tau^{2}-v_{0} \tau\right)+\frac{1}{2} \delta\left(\Delta y-\frac{1}{2} a \tau^{2}+v_{0} \tau\right),
$$

where $\delta(\cdot)$ represents the Dirac $\delta$-function.

To get the distribution of $P(y, t)$, the PDF of the Lévy walk particle arriving at physical coordinate $y$ at time $t$, it is a good choice to turn to the help of comoving coordinate since the distribution of the location after each deterministic motion in physical coordinate is difficult to describe. However, the relation between the displacement $\Delta x$ in comoving coordinate and $\Delta y$ in physical coordinate is clear, i.e., $\Delta y=d(t) \Delta x$, where $t$ is the overall observation time. Denote $\lambda(\triangle x \mid t ; \tau)$ as the distribution of the displacement $\Delta x$ at time $t$ in comoving coordinate with running time $\tau$. According to (4), we have

$$
\begin{aligned}
\lambda(\triangle x \mid t ; \tau) & =d(t) \lambda(d(t) \triangle x ; \tau) \\
& =\frac{1}{2} d(t) \delta\left(d(t) \triangle x-\frac{1}{2} a \tau^{2}-v_{0} \tau\right)+\frac{1}{2} d(t) \delta\left(d(t) \Delta x-\frac{1}{2} a \tau^{2}+v_{0} \tau\right) \\
& =\frac{1}{2} \delta\left(\triangle x-\frac{\frac{1}{2} a \tau^{2}+v_{0} \tau}{d(t)}\right)+\frac{1}{2} \delta\left(\Delta x-\frac{\frac{1}{2} a \tau^{2}-v_{0} \tau}{d(t)}\right) .
\end{aligned}
$$

The reason for the holding of the equalities is that the scale factor of the non-static medium we considered here is described by the exponential function $d(t)=\exp (H t)$ or power-law function $d(t)=\left(\frac{t+t_{0}}{t_{0}}\right)^{\beta}$, which implies $d(t)>0$ at any time. On the other hand, let $W(x, t)$ denote the PDF that the particle moves in comoving coordinate. Then

$$
P(y, t)=\frac{1}{d(t)} W\left(\frac{y}{d(t)}, t\right)
$$

on account of (2).

We are going to derive the master equation of Lévy walk particles moving under the action of a constant force in comoving coordinate with the standard formalism for the ordinary Lévy walk model. Introducing $q(x, t)$ to denote the PDF that the particle just arrives at $x$ at time $t$ in comoving coordinate, one yields

$$
q(x, t)=\int_{-\infty}^{\infty} d \Delta x \int_{0}^{t} q(x-\Delta x, t-\tau) \phi(\tau) \lambda(\Delta x \mid t ; \tau) d \tau+P_{0}(x) \delta(t) .
$$


Moving from the position $x-\Delta x$ at previous time $t-\tau$ to $x$ at $t$ implies that the particle needs to make a displacement $\Delta x$ in the running time $\tau$. The second term on the r.h.s. represents the initial distribution.

To calculate the PDF $W(x, t)$ at a given point in space and time, it is necessary to connect the flux at the renewal point to the density at the given point, i.e.,

$$
W(x, t)=\int_{-\infty}^{\infty} d \triangle x \int_{0}^{t} q(x-\triangle x, t-\tau) \psi(\tau) \lambda(\Delta x \mid t ; \tau) d \tau,
$$

where the definition of $\psi(\tau)$ is given in (5). After taking Laplace transform defined as $\hat{g}(s)=\mathcal{L}_{t}\{g(t)\}(s)=\int_{0}^{\infty} e^{-s t} g(t) d t$, we get

$$
\hat{\psi}(s)=\frac{1-\hat{\phi}(s)}{s} .
$$

The particle arrives at renewal point $x-\Delta x$ at time $t-\tau$, and it keeps surviving for time $\tau$ and the corresponding displacement for the running time $\tau$ is $\Delta x$ to make sure it locates at position $x$ at time $t$.

Plugging (7) into (9) and (10), we obtain

$$
\begin{aligned}
q(x, t)= & \frac{1}{2} \int_{0}^{t} q\left(x-\frac{\frac{1}{2} a \tau^{2}+v_{0} \tau}{d(t)}, t-\tau\right) \phi(\tau) d \tau \\
& +\frac{1}{2} \int_{0}^{t} q\left(x-\frac{\frac{1}{2} a \tau^{2}-v_{0} \tau}{d(t)}, t-\tau\right) \phi(\tau) d \tau+P_{0}(x) \delta(t)
\end{aligned}
$$

and

$$
\begin{aligned}
W(x, t)= & \frac{1}{2} \int_{0}^{t} q\left(x-\frac{\frac{1}{2} a \tau^{2}+v_{0} \tau}{d(t)}, t-\tau\right) \psi(\tau) d \tau \\
& +\frac{1}{2} \int_{0}^{t} q\left(x-\frac{\frac{1}{2} a \tau^{2}-v_{0} \tau}{d(t)}, t-\tau\right) \psi(\tau) d \tau .
\end{aligned}
$$

The normalization of $W(x, t)$ can be verified by the traditional method of integral transform. Since $\int_{-\infty}^{\infty} \mathcal{L}_{s}^{-1}\{\widehat{W}(x, s)\}(t) d x=\mathcal{L}_{s}^{-1}\left\{\left.\widehat{\widetilde{W}}(k, s)\right|_{k=0}\right\}(t)$, we take Fourier transform defined as $\tilde{f}(k)=\mathcal{F}_{x}\{f(x)\}(k)=\int_{-\infty}^{\infty} e^{-i k x} f(x) d x$ on (12) and (13) and make $k=0$. Further taking Laplace transform, there exists

$$
\left.\widehat{\widetilde{W}}(k, s)\right|_{k=0}=\left.\widehat{\widetilde{q}}(k, s)\right|_{k=0} \hat{\psi}(s)=\frac{1}{1-\hat{\phi}(s)} \frac{1-\hat{\phi}(s)}{s}=\frac{1}{s},
$$

where verifies the normalization of $W(x, t)$. However, this widely used method does not work well for analyzing the other statistical observables of our model because of its characteristics of time and space coupling. In the next section we will use Hermite orthogonal polynomials to approach the PDF in comoving coordinate.

\section{Hermite Polynomials Approximation to Lévy Walk in Constant Force Field in Non-static Medium}

In this section we apply Hermite polynomials to deal with the problem of spatiotemporally coupling appeared in comoving coordinate. The Hermite polynomials form an orthogonal 
basis of the Hilbert space with the inner product $\langle f, g\rangle=\int_{-\infty}^{\infty} f(x) \bar{g}(x) e^{-x^{2}} d x$ [3]. We assume that in Hilbert space $q(x, t)$ and $W(x, t)$ can be, respectively, expressed as

$$
\begin{aligned}
q(x, t) & =\sum_{n=0}^{\infty} H_{n}(x) T_{n}(t) e^{-x^{2}}, \\
W(x, t) & =\sum_{n=0}^{\infty} H_{n}(x) R_{n}(t) e^{-x^{2}},
\end{aligned}
$$

where $H_{n}(x), n=0,1, \ldots$, represent the Hermite polynomials, and $\left\{T_{n}(t)\right\}$ and $\left\{R_{n}(t)\right\}$ are a series of functions with respect to $t$ to be determined, respectively.

The initial distribution of the particle is assumed to be a Dirac-delta function, i.e., $P_{0}(x)=\delta(x)$. Inserting (14) and (15) into (12) and (13), respectively, the iteration relation between $\widehat{T}_{m}(s)$ and the relation between $\widehat{R}_{m}(s)$ and $\widehat{T}_{m}(s)$ can be written after taking Laplace transform, respectively, as

$$
\begin{aligned}
\sqrt{\pi} 2^{m} m ! \widehat{T}_{m}(s)= & \frac{1}{2} \sum_{k=0}^{m} \frac{2^{k} \sqrt{\pi} m !}{(m-k) !} \times \mathcal{L}_{t}\left\{( \frac { 1 } { d ( t ) } ) ^ { m - k } \mathcal { L } _ { s } ^ { - 1 } \left[\widehat{T}_{k}(s) \mathcal{L}_{\tau}[\phi(\tau)\right.\right. \\
& {\left.\left.\left.\left[\left(a \tau^{2}-2 v_{0} \tau\right)^{m-k}+\left(a \tau^{2}+2 v_{0} \tau\right)^{m-k}\right]\right](s)\right](t)\right\}(s)+H_{m}(0) }
\end{aligned}
$$

and

$$
\begin{aligned}
\sqrt{\pi} 2^{m} m ! \widehat{R}_{m}(s)= & \frac{1}{2} \sum_{k=0}^{m} \frac{2^{k} \sqrt{\pi} m !}{(m-k) !} \times \mathcal{L}_{t}\left\{\left(\frac{1}{d(t)}\right)^{m-k} \mathcal{L}_{s}^{-1}\right. \\
& {\left.\left[\widehat{T}_{k}(s) \mathcal{L}_{\tau}\left[\psi(\tau)\left[\left(a \tau^{2}-2 v_{0} \tau\right)^{m-k}+\left(a \tau^{2}+2 v_{0} \tau\right)^{m-k}\right]\right](s)\right](t)\right\}(s) . }
\end{aligned}
$$

The details of derivation are shown in Appendix B.

Some important statistics of the process are determined by a series of functions to be determined, e.g., $\left\{T_{n}(t)\right\}$ and $\left\{R_{n}(t)\right\}$. For example, in [73], the authors state that the longtime asymptotic behaviors of the moments of the particle's position have a close relation with the derivative of the PDF $\widetilde{W}(k, s)$ in Fourier-Laplace space, which reads

$$
\left\langle\hat{x}^{m}(s)\right\rangle=\left.(i)^{m} \frac{\partial^{m}}{\partial k^{m}} \widetilde{\widetilde{W}}(k, s)\right|_{k=0} .
$$

It is quite necessary to derive the expression of $\widetilde{\widetilde{W}}(k, s)$. Starting from (15) and (63), $W(x, t)$ can be written as

$$
W(x, t)=\sum_{n=0}^{\infty}(-1)^{n} \frac{d^{n}}{d x^{n}} e^{-x^{2}} R_{n}(t) .
$$

Taking Fourier transform $x \rightarrow k$ and Laplace transform $t \rightarrow s$ on (19), it yields

$$
\widehat{\widetilde{W}}(k, s)=\sum_{n=0}^{\infty} \sqrt{\pi}(-i k)^{n} e^{-\frac{k^{2}}{4}} \widehat{R}_{n}(s) .
$$

Combining (18) with (20), we present the asymptotic expressions of the first two moments of this process in comoving coordinate, after sufficiently long time, which, respectively, behave as

$$
\langle\hat{x}(s)\rangle=\left.i \frac{\partial}{\partial k} \widehat{\widetilde{W}}(k, s)\right|_{k=0}=\sqrt{\pi} \widehat{R}_{1}(s)
$$


and

$$
\left\langle\hat{x}^{2}(s)\right\rangle=-\left.\frac{\partial^{2}}{\partial k^{2}} \widehat{\widetilde{W}}(k, s)\right|_{k=0}=\frac{\sqrt{\pi}}{2} \widehat{R}_{0}(s)+2 \sqrt{\pi} \widehat{R}_{2}(s) .
$$

In addition,

$$
\left.\widehat{\widetilde{W}}(k, s)\right|_{k=0}=\sqrt{\pi} \widehat{R}_{0}(s) .
$$

Notably, we can get the values of $\widehat{R}_{0}(s), \widehat{R}_{1}(s)$, and $\widehat{R}_{2}(s)$ through (16) and (17). Respectively taking $m=0,1,2$ in (16) and (17) leads to

$$
\begin{aligned}
\widehat{T}_{0}(s) & =\frac{1}{\sqrt{\pi}(1-\hat{\phi}(s))} \\
\widehat{R}_{0}(s) & =\frac{1}{s \sqrt{\pi}} \\
2 \widehat{T}_{1}(s) & =\mathcal{L}_{t}\left\{\frac{1}{d(t)} \mathcal{L}_{s}^{-1}\left[a \hat{\phi}^{\prime \prime}(s) \widehat{T}_{0}(s)\right](t)\right\}(s)+2 \widehat{T}_{1}(s) \hat{\phi}(s), \\
2 \widehat{R}_{1}(s) & =\mathcal{L}_{t}\left\{\frac{1}{d(t)} \mathcal{L}_{s}^{-1}\left[a \hat{\psi}^{\prime \prime}(s) \widehat{T}_{0}(s)\right](t)\right\}(s)+2 \widehat{T}_{1}(s) \hat{\psi}(s),
\end{aligned}
$$

and

$$
\begin{aligned}
8 \sqrt{\pi} \widehat{T}_{2}(s)= & 4 a \sqrt{\pi} \mathcal{L}_{t}\left\{\frac{1}{d(t)} \mathcal{L}_{s}^{-1}\left[\widehat{T}_{1}(s) \hat{\phi}^{\prime \prime}(s)\right](t)\right\}(s)+8 \sqrt{\pi} \hat{\phi}(s) \widehat{T}_{2}(s)-2 \\
& +\sqrt{\pi} \mathcal{L}_{t}\left\{\frac{1}{d^{2}(t)} \mathcal{L}_{s}^{-1}\left[\widehat{T}_{0}(s)\left(a^{2} \hat{\phi}^{4}(s)+4 v_{0}^{2} \hat{\phi}^{\prime \prime}(s)\right)\right](t)\right\}(s), \\
8 \widehat{R}_{2}(s)= & 4 a \mathcal{L}_{t}\left\{\frac{1}{d(t)} \mathcal{L}_{s}^{-1}\left[\widehat{T}_{1}(s) \hat{\psi}^{\prime \prime}(s)\right](t)\right\}(s)+8 \hat{\psi}(s) \widehat{T}_{2}(s) \\
& +\mathcal{L}_{t}\left\{\frac{1}{d^{2}(t)} \mathcal{L}_{s}^{-1}\left[\widehat{T}_{0}(s)\left(a^{2} \hat{\psi}^{4}(s)+4 v_{0}^{2} \hat{\psi}^{\prime \prime}(s)\right)\right](t)\right\}(s) .
\end{aligned}
$$

We verify the normalization of $W(x, t)$ with the help of $\widehat{R}_{0}(s)=\frac{1}{s \sqrt{\pi}}$ since $\mathcal{L}_{s}^{-1}\left\{\int_{-\infty}^{\infty} \widehat{W}(x, s) d x\right\}(t)=\mathcal{L}_{s}^{-1}\left\{\left.\widehat{\widetilde{W}}(k, s)\right|_{k=0}\right\}(t)=\mathcal{L}_{s}^{-1}\left\{\sqrt{\pi} \widehat{R}_{0}(s)\right\}(t)=\mathcal{L}_{s}^{-1}$ $\left\{\frac{1}{s}\right\}(t)=1$. Obviously, the PDF $P(y, t)$ in physical coordinate is also normalized. Furthermore, in view of (2), the asymptotic expressions of the first two moments in physical coordinate are

$$
\langle y(t)\rangle=d(t)\langle x(t)\rangle
$$

and

$$
\left\langle y^{2}(t)\right\rangle=d^{2}(t)\left\langle x^{2}(t)\right\rangle
$$

In the next section, we are going to analyze the long-time asymptotic behaviours of the first two moments in both coordinates for some specific running time PDFs $\phi(t)$ and scale factors $d(t)$. 


\section{Dynamical Behaviours of Lévy Walk Under the Action of an External Constant Force in Non-static Medium}

\subsection{Exponentially Distributed Running Time}

In this subsection the PDF of running time is assumed to be exponential distribution, i.e., $\phi(\tau)=\lambda e^{-\lambda \tau}$ with $\lambda>0$. As is shown in [73], the diffusion of classical Lévy walk in static medium is normal diffusion when the running time PDF obeys exponential distribution. We are going to explore the combined effect of constant force and non-static medium on the dynamics of Lévy walk.

\subsubsection{Exponential Scale Factor}

Assume that the scale factor of the non-static medium in this part is described by the exponential form,

$$
d(t)=\exp (H t)
$$

with the "Hubble constant" $H$. In the context of cosmology $[52,56]$, this type of non-static medium is caused by dark energy. Apparently $H>0$ means an expanding medium whereas $H<0$ represents a contracting one.

Combining with (21) and (22), the first two moments in comoving coordinate are derived by plugging the expressions of $\phi(\tau)$ and $d(t)$ into (24)-(28), which respectively read as

$$
\langle x(t)\rangle \sim \begin{cases}\frac{a \lambda}{H(H+\lambda)^{2}}, & \text { if } H>0, \\ \frac{a(H-\lambda)}{H \lambda^{2}} e^{-H t}, & \text { if } H<0,\end{cases}
$$

and

$$
\left\langle x^{2}(t)\right\rangle \sim \begin{cases}\frac{a^{2} \lambda\left(3 H^{3}+10 H^{2} \lambda+7 H \lambda^{2}+\lambda^{3}\right)+H \lambda(H+\lambda)^{2}(2 H+\lambda)^{2} v_{0}^{2}}{H^{2}(H+\lambda)^{2}(2 H+\lambda)^{4}}, & \text { if } H>0, \\ \frac{(2 H-\lambda)\left[a^{2}\left(3 H^{3}-6 H^{2} \lambda+3 H \lambda^{2}-\lambda^{3}\right)+H(H-\lambda)^{2} \lambda^{2} v_{0}^{2}\right]}{H^{2}(H-\lambda)^{2} \lambda^{4}} e^{-2 H t}, & \text { if } H<0 .\end{cases}
$$

Correspondingly, the first two moments in physical coordinate can be straightforwardly obtained in terms of (29) and (30) after sufficiently long time, which respectively read as

$$
\langle y(t)\rangle \sim \begin{cases}\frac{a \lambda}{H(H+\lambda)^{2}} e^{H t}, & \text { if } H>0, \\ \frac{a(H-\lambda)}{H \lambda^{2}}, & \text { if } H<0,\end{cases}
$$

and

$$
\left\langle y^{2}(t)\right\rangle \sim \begin{cases}\frac{a^{2} \lambda\left(3 H^{3}+10 H^{2} \lambda+7 H \lambda^{2}+\lambda^{3}\right)+H \lambda(H+\lambda)^{2}(2 H+\lambda)^{2} v_{0}^{2}}{H^{2}(H+\lambda)^{2}(2 H+\lambda)^{4}} e^{2 H t}, & \text { if } H>0, \\ \frac{(2 H-\lambda)\left[a^{2}\left(3 H^{3}-6 H^{2} \lambda+3 H \lambda^{2}-\lambda^{3}\right)+H(H-\lambda)^{2} \lambda^{2} v_{0}^{2}\right]}{H^{2}(H-\lambda)^{2} \lambda^{4}}, & \text { if } H<0 .\end{cases}
$$

The results of the first two moments in both coordinates are verified in Figs. 2 and 3. For $H>0$, the first two moments in comoving coordinate tend to be a constant whereas grow exponentially without limit in physical coordinate for sufficiently long time. In contrast, the first two moments in comoving coordinate exponentially increase with respect to time $t$ whereas are fixed in physical coordinate for long time limit for $H<0$. In addition, the average moments in both coordinates have no relation with $v_{0}$. However, the MSDs in both coordinates rely on the value of $v_{0}$. Moreover, by taking $a=0$, we recover the corresponding results of the first two moments of pure Lévy walk in non-static medium in both coordinates 

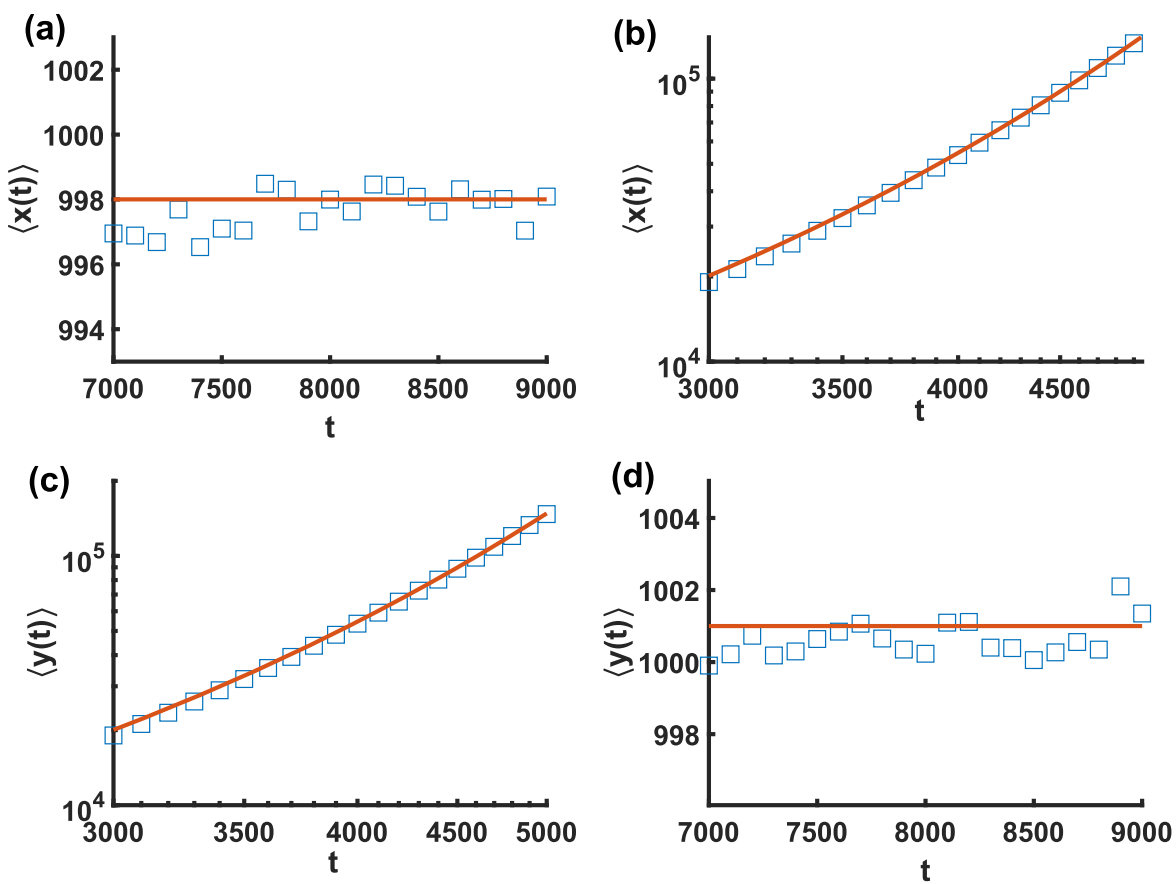

Fig. 2 Numerical simulations of the average moments of Lévy walk under the action of constant force in non-static medium with exponential scale factor by sampling over $10^{4}$ realizations. The running time PDF of Lévy walk behaves as exponential distribution $\phi(\tau)=\lambda e^{-\lambda \tau}$ with $\lambda=1$. The other parameters are $v_{0}=1$, $a=1$, and $x_{0}=y_{0}=0$. For (a) and (c), we take $H=10^{-3}$. For (b) and (d), $H=-10^{-3}$

[75]. The average moments in [75] appear as 0 in both coordinates whether the medium expands or contracts. We conclude that the presence of external force changes the symmetry of the position distribution. Furthermore, compared with the first two moments of Lévy walk under the action of constant force in static medium [74] which respectively appear as $\langle y(t)\rangle \sim t$ and $\left\langle y^{2}(t)\right\rangle \sim t^{2}$, it can be seen that the deterministic motion induced by non-static medium dominates the overall process.

A stationary distribution can be eventually reached in comoving or physical coordinate when the medium exponentially expands $H>0$ or contracts $H<0$. In the following we analyze the stationary distribution $P^{s t}(x)$ or $P^{s t}(y)$ of Lévy walk in constant force field in comoving or physical coordinate by numerical simulations. For Lévy walk with $\phi(\tau)=\lambda e^{-\lambda \tau}$ moving in constant force field and a positive $H$ in comoving coordinate, as shown in Fig. 4a, the phenomena of bimodal-to-unimodal crossovers can be observed by increasing the value of $\lambda$. However, the unimodality of the stationary distribution $P^{s t}(x)$ does not alter if we respectively change the value of $H, v_{0}, a$. To be more specific, Fig. 4(b) indicates that changing the value of $H$ does not mean the conversion of the stationary distribution $P^{s t}(x)$ between unimodal and bimodal, and increasing $H$ can only taper $P^{s t}(x)$. Similarly, Fig. $4 \mathrm{c}$ states that increasing the value of $v_{0}$ can only flatten $P^{s t}(x)$, so as $a$ does in Fig. 4d. Interestingly, for Lévy walk with a negative $H$ in physical coordinate, the unimodal property of $P^{s t}(y)$ can not be affected by the variation of $\lambda, H, v_{0}, a$, respectively. Specially, from Fig. 5a, b the stationary distribution is gradually narrowed with the increase of $\lambda$ and $|H|$ while the stationary distribution is flattened as $v_{0}$ and $a$ increase which has been shown 

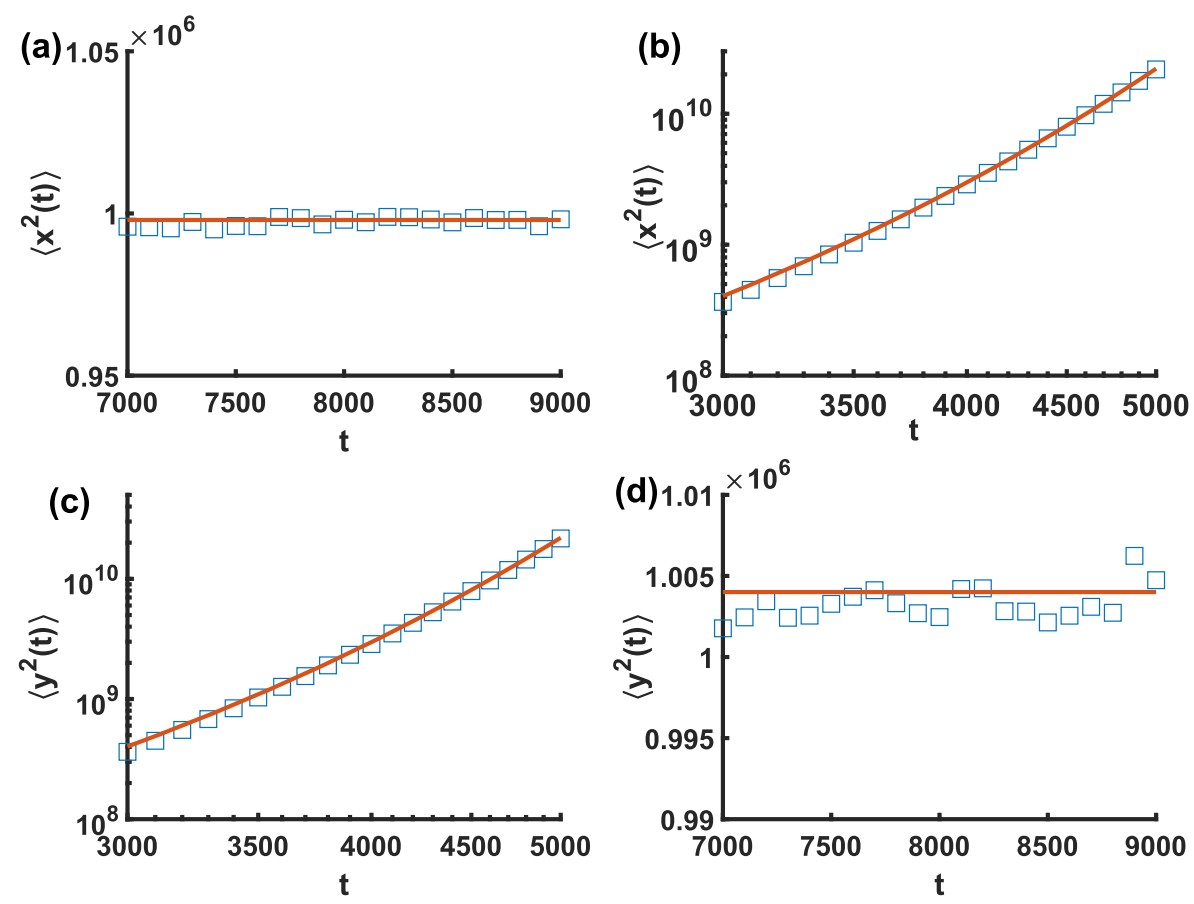

Fig. 3 Numerical simulations of MSDs of Lévy walk under the action of constant force in non-static medium with exponential scale factor in both coordinates by sampling over $10^{4}$ realizations. The other parameters are same with the ones of Fig. 2

in Fig. 5c, d. Notably, the effect of $\lambda$ turns out to be completely different in both coordinates; the bimodal or monomodal state of stationary distribution $P^{s t}(x)$ depends on the value of $\lambda$ in comoving coordinate while decreasing the value of $\lambda$ in physical coordinate only flattens the stationary distribution $P^{s t}(y)$.

Kurtosis is another important statistic which describes the tails of the stationary PDF. Here, we only consider the kurtosis of stationary distribution in physical coordinate for $H<0$. It is defined as

$$
K=\frac{\left\langle[y(t)-\mu]^{4}\right\rangle}{\left\langle[y(t)-\mu]^{2}\right\rangle^{2}},
$$

where $\mu$ represents the average moment in exponential contraction medium, i.e., $\mu=\frac{a(H-\lambda)}{H \lambda^{2}}$. We further expand Kurtosis as

$$
K=\frac{\left\langle y^{4}(t)\right\rangle-4 \mu\left\langle y^{3}(t)\right\rangle+6 \mu^{2}\left\langle y^{2}(t)\right\rangle-3 \mu^{4}}{\left(\left\langle y^{2}(t)\right\rangle-\mu^{2}\right)^{2}},
$$

which relies on the first four moments in physical coordinate. With the help of (8), we use the moments in comoving coordinate to express the value of Kurtosis in (37). The equivalent expression of (37) is

$$
K=\frac{\left\langle x^{4}(t)\right\rangle-4 \mu_{x}\left\langle x^{3}(t)\right\rangle+6 \mu_{x}^{2}\left\langle x^{2}(t)\right\rangle-3 \mu_{x}^{4}}{\left(\left\langle x^{2}(t)\right\rangle-\mu_{x}^{2}\right)^{2}},
$$



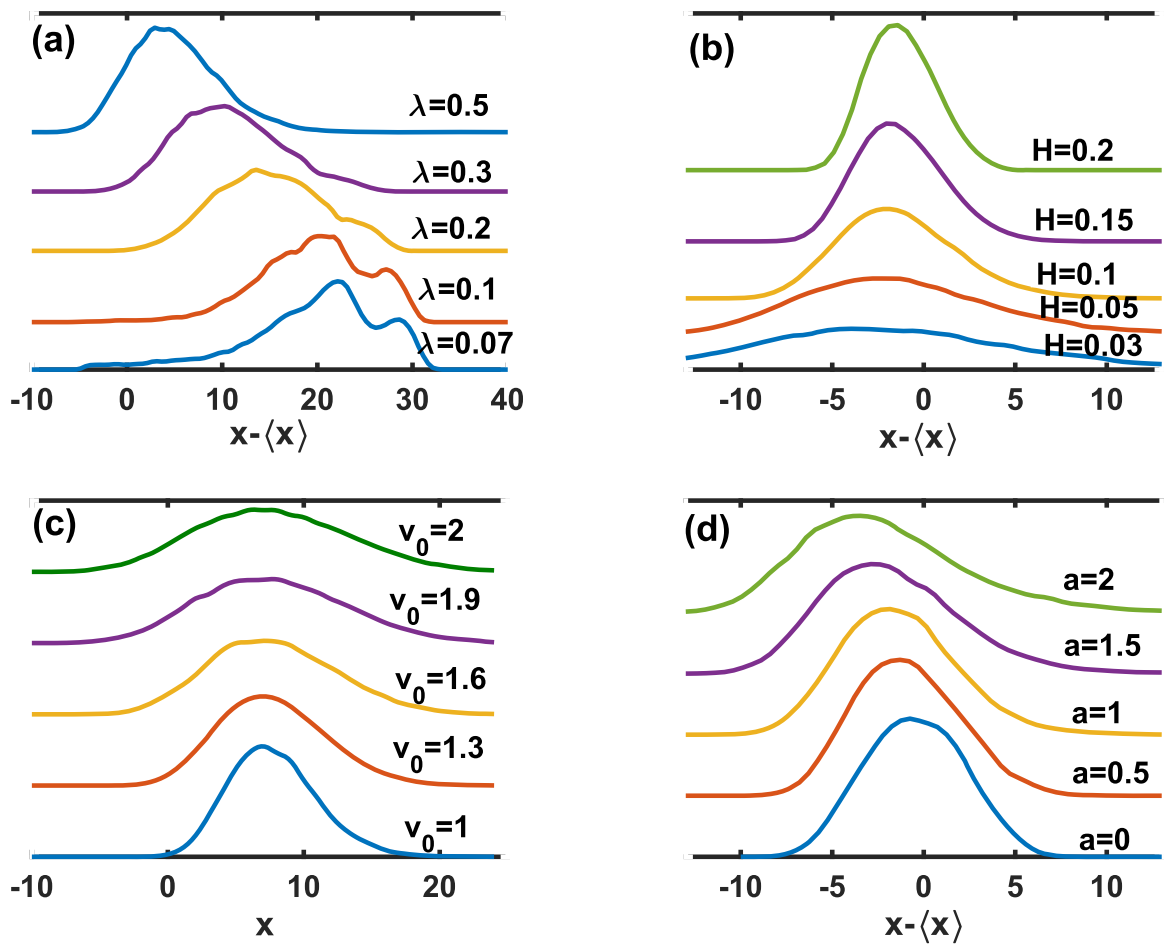

Fig. 4 Stationary PDFs of Lévy walk with $\phi(\tau)=\lambda e^{-\lambda \tau}$ in constant force field in comoving coordinate and exponentially expanding scale factor by sampling over $2 \times 10^{4}$ realizations. Here we assume $x_{0}=0$. For panel (a), we take $H=0.1, a=v_{0}=1$; for panel (b), $a=v_{0}=\lambda=1$; for panel (c), $a=\lambda=1, H=0.1$; for panel $(\mathbf{d}), H=0.1, \lambda=v_{0}=1$

where the indicator $x$ in $\mu_{x}$ means the average moment in comoving coordinate, i.e., $\mu_{x}=$ $\frac{a(H-\lambda)}{H \lambda^{2}} e^{-H t}$. In the following, we turn to the moments in comoving coordinate. According to (18) and (20), the moments we cared can be represented by a series of functions $\widehat{R}_{i}(s)$ after some calculations, i.e.,

$$
\left\langle\hat{x}^{3}(s)\right\rangle=\frac{3}{2} \sqrt{\pi} \widehat{R}_{1}(s)+6 \sqrt{\pi} \widehat{R}_{3}(s)
$$

and

$$
\left\langle\hat{x}^{4}(s)\right\rangle=\frac{3}{4} \sqrt{\pi} \widehat{R}_{0}(s)+6 \sqrt{\pi} \widehat{R}_{2}(s)+24 \sqrt{\pi} \widehat{R}_{4}(s) .
$$

In order to get the values of $\widehat{R}_{i}(s)(i=0,1,2,3,4)$, besides (24)-(28), we respectively take $m=3,4$ in (16) and (17) and derive the recurrence relation between $\widehat{T}_{i}(s)$ and $\widehat{R}_{i}(s)$ and the iteration relation between $\widehat{T}_{i}(s)(\mathrm{i}=0,1,2,3,4)$. Combining with the concrete expressions of running time as well as scale factor, the corresponding first four moments in comoving coordinates can be got. Plugging them into Eq. (38), we get the long-time asymptotic expression of Kurtosis shown in (1) in exponential contraction medium.

The value of Kurtosis has a close relation with the inverse average of exponentially distributed running time $\lambda$, the Hubble constant $H$, constant acceleration $a$, as well as the initial speed of each step $v_{0}$, being verified in Fig. 6. At first for any fixed $H, v_{0}, a$, we consider $K$ 

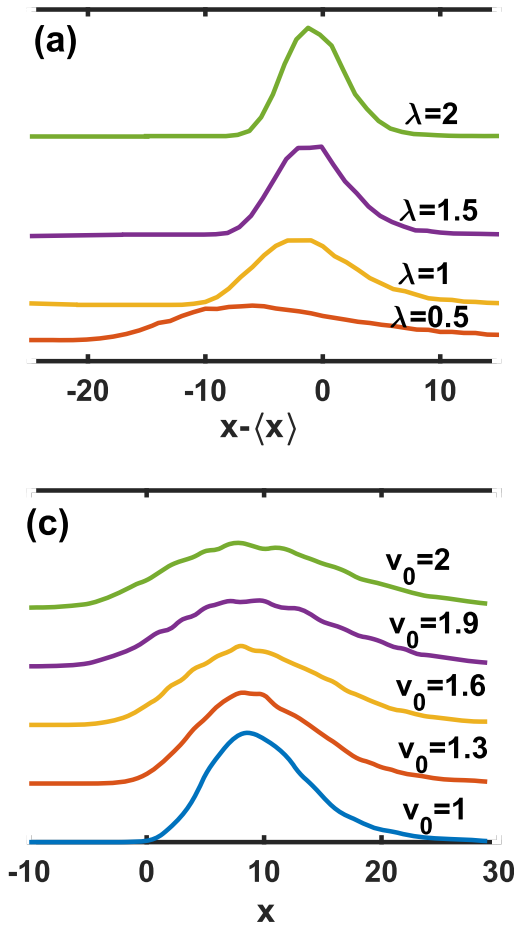
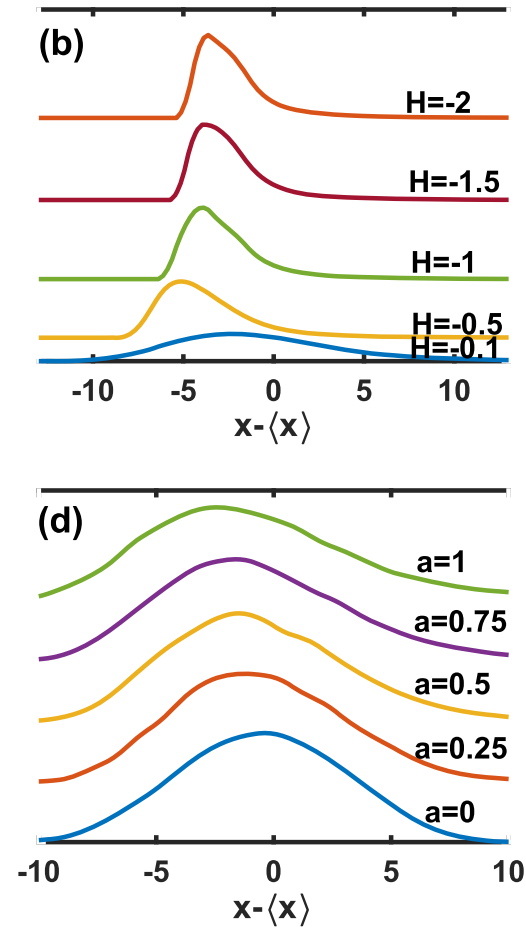

Fig. 5 Stationary PDFs of Lévy walk with $\phi(\tau)=\lambda e^{-\lambda \tau}$ in constant force field in physical coordinate and exponentially contracting scale factor by sampling over $2 \times 10^{4}$ realizations. Here we assume $y_{0}=0$. For panel (a), we take $H=-0.1, a=v_{0}=1$; for panel (b), $a=v_{0}=\lambda=1$; for panel (c), $a=\lambda=1$, $H=-0.1$; for panel $(\mathbf{d}), H=-0.1, \lambda=v_{0}=1$

as a function of $\lambda$. For small $\lambda$, after sufficiently long time, the stationary PDF is leptokurtic since $K_{\lambda \rightarrow 0} \sim 87.72$. However, for large $\lambda$, a Gaussian PDF emerges since $K_{\lambda \rightarrow \infty} \sim 3$. The above results are consistent with our simulation results in Fig. 6a. Next we consider $K$ as a function of $v_{0}$ for any fixed $H, \lambda, a$; it can be found that the limit value of Kurtosis for small or large $v_{0}$ relies on $H, \lambda$. The same conclusion holds on if we consider $K$ as a function of $a$ for any fixed $H, \lambda, v_{0}$. Surprisingly, we discover that $K_{v_{0} \rightarrow 0}=K_{a \rightarrow \infty}$ and $K_{v_{0} \rightarrow \infty}=K_{a \rightarrow 0}$ (see (75) and (76) for details). Finally, as above, we consider $K$ as a function of $H$ for any fixed $v_{0}, \lambda, a$; the PDF converges to the Gaussian value $K=3$ for small $|H|$. Moreover, we have the limit of $K$ for large $|H|$ from (1), i.e., $K_{H \rightarrow-\infty} \sim \frac{3\left(731 a^{4}+316 a^{2} \lambda^{2} v_{0}^{2}+8 \lambda^{4} v_{0}^{4}\right)}{\left(5 a^{2}+2 \lambda^{2} v_{0}^{2}\right)^{2}}$, which has relevance to $a, \lambda, v_{0}$.

\subsubsection{Power-Law Scale Factor}

We focus on the case that the scale factor of the non-static medium is power-law distribution

$$
d(t)=\left(\frac{t+t_{0}}{t_{0}}\right)^{\beta}
$$



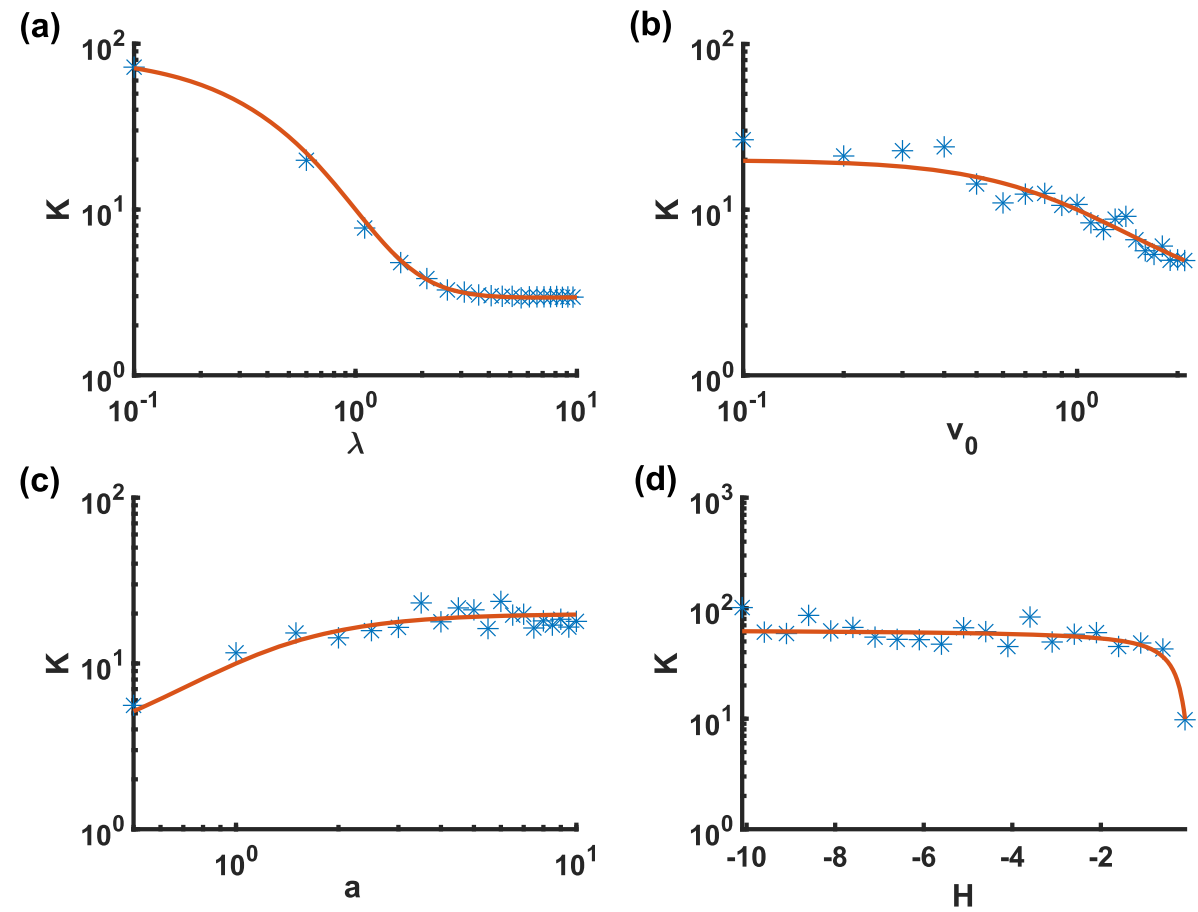

Fig. 6 Numerical simulations of kurtosis of Lévy walk under the action of constant force in non-static medium with exponential contraction scale factor by sampling over $5 \times 10^{4}$ realizations. The running time PDF of Lévy walk behaves as exponential distribution $\phi(\tau)=\lambda e^{-\lambda \tau}$. The initial position in physical coordinate is $y_{0}=0$. The figure $\mathbf{a}$ is for $K$ versus $\lambda$ for fixed $H=-0.1, v_{0}=1, a=1$; the figure $\mathbf{b}$ is for $K$ versus $v_{0}$ for fixed $H=-0.1, \lambda=a=1$; the figure $\mathbf{c}$ is for $K$ versus $a$ for fixed $H=-0.1, \lambda=v_{0}=1$; the figure $\mathbf{d}$ is for $K$ versus $H$ for fixed $a=\lambda=v_{0}=1$. The solid lines are the theoretical results shown in (1)

where $t$ means the time lapsed since the initial time $t_{0}$. The value of $\beta>0$ corresponds to an expanding medium since $\dot{d}(t)>0$ while the value of $\beta<0$ corresponds to a contracting medium.

Plugging the power-law scale factor $d(t)$ and the exponentially distributed running time $\phi(\tau)$ into (25)-(28) and combining with (21) and (22), after sufficiently long time, one has

$$
\langle x(t)\rangle \sim \begin{cases}\frac{a t_{0}^{\beta}}{\lambda(1-\beta)} t^{1-\beta}, & \text { if } \beta<1, \\ \frac{a t_{0}\left(-1+e^{\lambda t_{0}}\left(\lambda t_{0}-1\right) \Gamma\left(0, \lambda t_{0}\right)+\ln (t)-\ln \left(t_{0}\right)\right)}{\lambda}, & \text { if } \beta=1, \\ \frac{a t_{0}\left(-1+\frac{1}{-1+\beta}+e^{\lambda t}\left(\lambda t_{0}-2+\beta\right) E_{\beta}\left(\lambda t_{0}\right)\right)}{\lambda}, & \text { if } \beta>1,\end{cases}
$$

and

$$
\left\langle x^{2}(t)\right\rangle \sim \begin{cases}\frac{a^{2} t_{0}^{2 \beta}}{(\beta-1)^{2} \lambda^{2}} t^{2-2 \beta}, & \text { if } \beta<1, \\ \frac{a^{2} t_{0}^{2}}{\lambda^{2}}(\gamma+\ln (t))^{2}, & \text { if } \beta=1, \\ C_{0}, & \text { if } \beta>1,\end{cases}
$$

where $E_{n}(x)$ is the exponential integral function defined as $E_{n}(x)=\int_{1}^{\infty} e^{-x t} / t^{n} d t$ and $\Gamma(a, b)$ is the incomplete Gamma function. In addition, $\gamma$ represents eulergamma constant, 

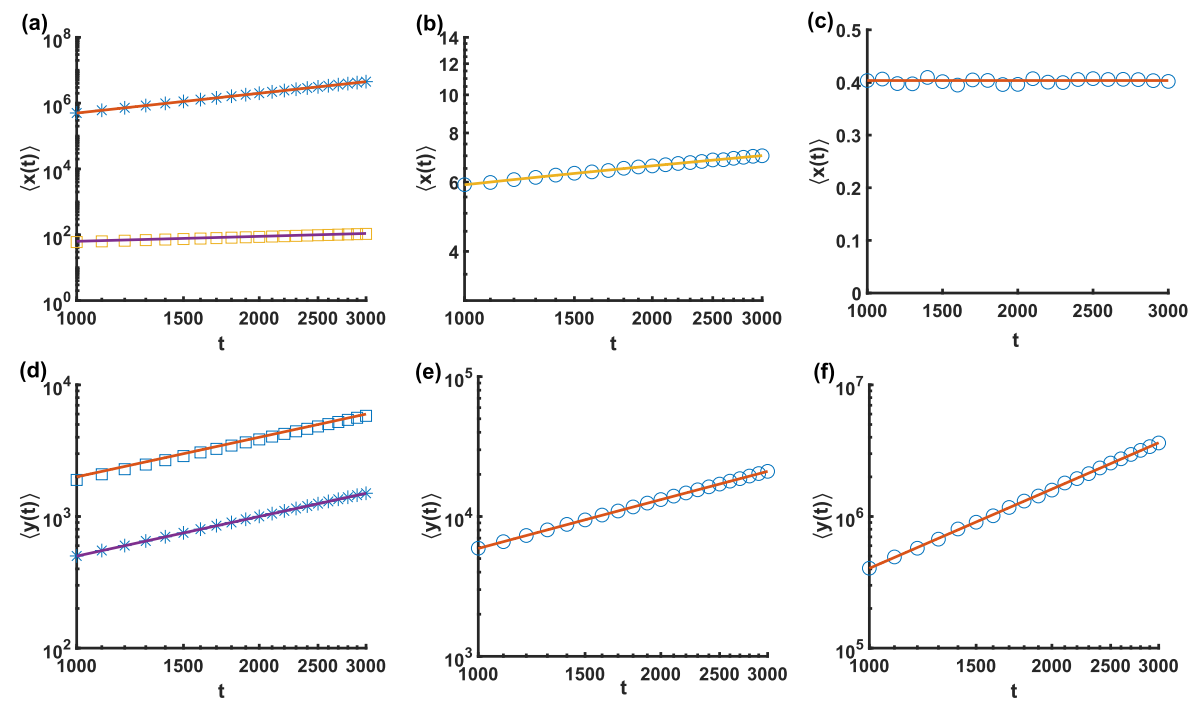

Fig. 7 Numerical simulations of the average moments of Lévy walk under the action of constant force in non-static medium with power-law scale factor by sampling over $10^{4}$ realizations. The running time PDF of Lévy walk behaves as exponential distribution $\phi(\tau)=\lambda e^{-\lambda \tau}$ with $\lambda=1$. The other parameters are $v_{0}=1$, $x_{0}=y_{0}=0, a=1$, as well as $t_{0}=1$. For (a) and (d), we respectively take $\beta=0.5$ (squares) and $\beta=-1$ (stars); for (b) and (e), $\beta=1$; for (c) and (f), $\beta=2$

approached by 0.577216 . However, it seems not easy to get the exact vule of $C_{0}$ in (43) because of the complicated calculations and approximations. Further, we find the long-time asymptotic behavior of the first two moments in physical coordinate, respectively reads

$$
\langle y(t)\rangle \sim \begin{cases}\frac{a}{\lambda(1-\beta)} t, & \text { if } \beta<1, \\ \frac{a\left(-1+e^{\lambda t_{0}}\left(\lambda t_{0}-1\right) \Gamma\left(0, \lambda t_{0}\right)+\ln (t)-\ln \left(t_{0}\right)\right)}{\lambda} t, & \text { if } \beta=1, \\ \frac{a\left(-1+\frac{1}{-1+\beta}+e^{\lambda t_{0}}\left(\lambda t_{0}-2+\beta\right) E_{\beta}\left(\lambda t_{0}\right)\right)}{\lambda t_{0}^{\beta-1}} t^{\beta}, & \text { if } \beta>1,\end{cases}
$$

and

$$
\left\langle y^{2}(t)\right\rangle \sim \begin{cases}\frac{a^{2}}{\lambda^{2}(1-\beta)^{2}} t^{2}, & \text { if } \beta<1, \\ \frac{a^{2}}{\lambda^{2}}(\gamma+\ln (t))^{2} t^{2}, & \text { if } \beta=1, \\ C_{0} t^{2 \beta}, & \text { if } \beta>1 .\end{cases}
$$

The results for the first two moments in both coordinates are verified in Figs. 7 and 8 . Since the diffusion in physical coordinate is superdiffusion, compared with the MSD of traditional free Lévy walk model in static medium which behaves as $\left\langle y^{2}(t)\right\rangle \sim t$, we conclude that the combined action of non-static medium and external constant potential accelerates the motion of particles. For $\beta<1$, the first two moments $\langle y(t)\rangle$ and $\left\langle y^{2}(t)\right\rangle$ in physical coordinate grow as $t$ and $t^{2}$, respectively, which are consistent with the results of Lévy walk under the action of external force in static medium in [74]. Therefore, the process is mainly driven by the external constant force and the non-static medium hardly affects the diffusion process. However, for $\beta>1$, the MSD $\left\langle y^{2}(t)\right\rangle$ in physical coordinate is approximate to $t^{2 \beta}$ (with logarithmic corrections in the marginal case $\beta=1$ ), which appears as same as the MSD of free Lévy walk in non-static medium [75]. Thus in this case, the spread of the walker is 

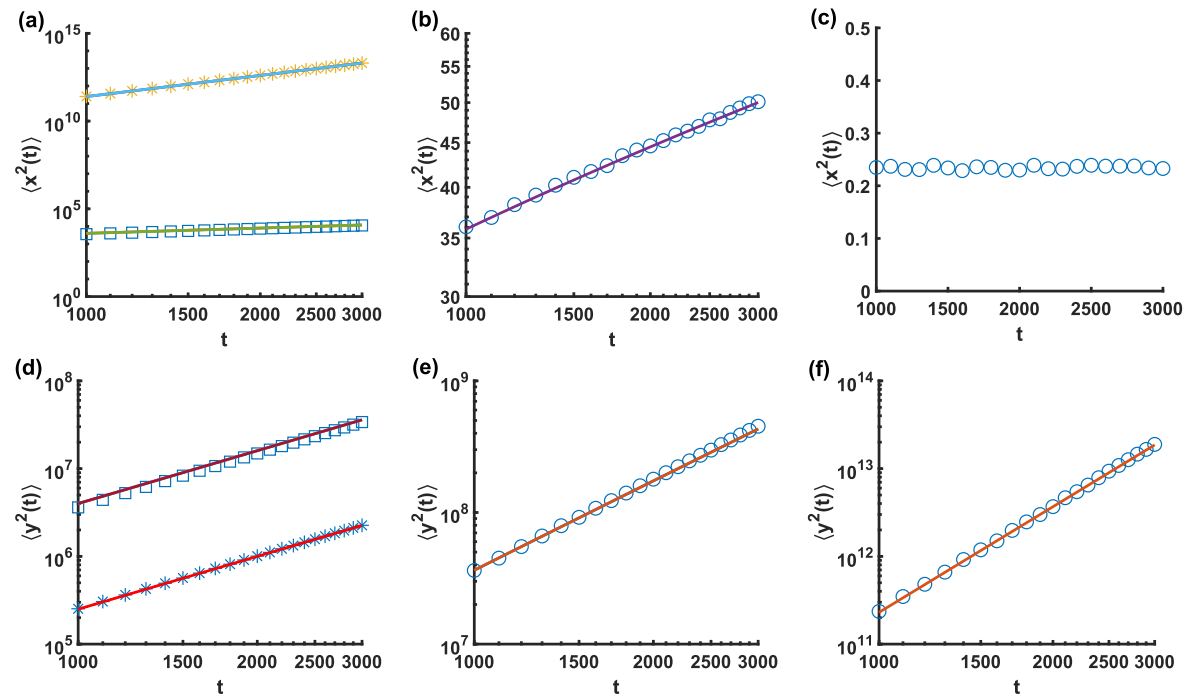

Fig. 8 Numerical simulations of MSDs of Lévy walk under the action of constant force in non-static medium with power-law scale factor by sampling over $10^{4}$ realizations. The running time PDF of Lévy walk behaves as exponential distribution $\phi(\tau)=\lambda e^{-\lambda \tau}$ with $\lambda=1$. The other parameters are same with the ones in Fig. 7

mainly driven by the non-static medium. Besides, after sufficiently long time, we find the initial velocity $v_{0}$ of each step does not affect the asymptotic forms of the first two moments in both coordinates.

\subsection{Power-Law Distributed Running Time}

Anomalous diffusion can be modeled by the traditional Lévy walk model in static medium when the PDF of running time behaves as Pareto distribution,

$$
\phi(\tau)=\frac{1}{\tau_{0}} \frac{\alpha}{\left(1+\tau / \tau_{0}\right)^{1+\alpha}},
$$

where $\tau_{0}>0$ and $\alpha>0$. As above, in this subsection we concentrate on analyzing the dynamical behaviors of Lévy walk under the combined action of an external force and the non-static medium on the condition that the running time is power-law distribution.

\subsubsection{Exponential Scale Factor}

Inserting the exponential scale factor $d(t)$ and power-law running time $\phi(\tau)$ into (25)-(28) and combining with (21) and (22), we calculate the first two moments in comoving coordinate for $H>0$ after sufficiently long time, which respectively behave as

$$
\langle x(t)\rangle \sim \frac{a \alpha\left(-1+\alpha+H \tau_{0}\right)-a \alpha e^{H \tau_{0}}\left((-1+\alpha) \alpha+2 \alpha H \tau_{0}+H^{2} \tau_{0}^{2}\right) E_{1+\alpha}\left(H \tau_{0}\right)}{2 H^{2}\left(-1+\alpha e^{H \tau_{0}} \tau_{0}^{\alpha} H^{\alpha} \Gamma\left(-\alpha, H \tau_{0}\right)\right)}
$$

and

$$
\left\langle x^{2}(t)\right\rangle \sim C
$$



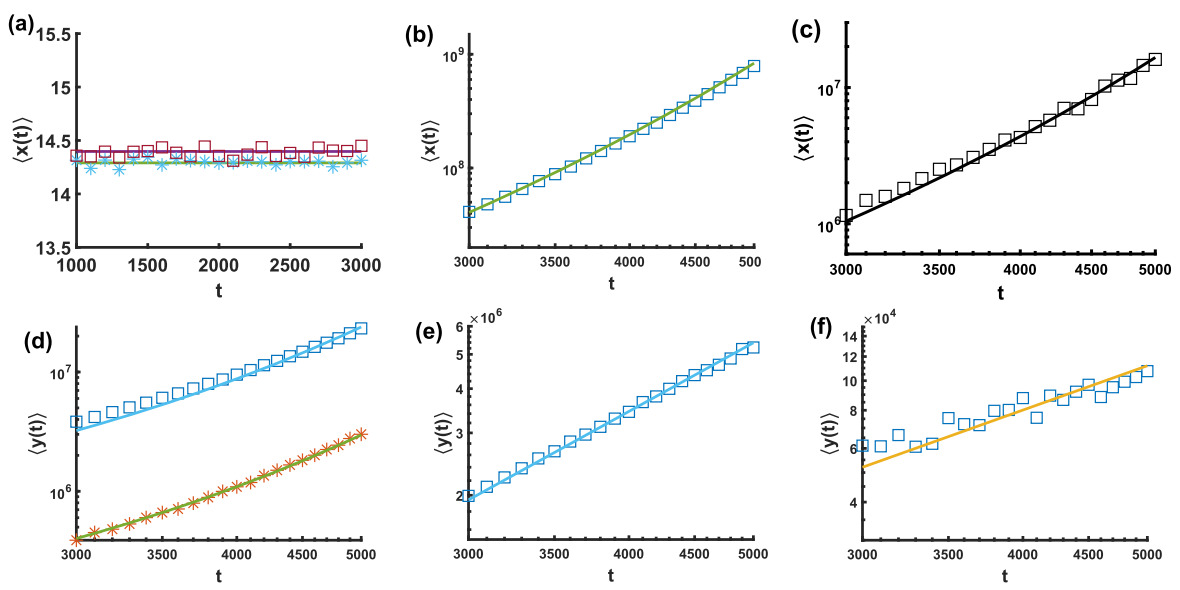

Fig. 9 Numerical simulations of the average moments of Lévy walk under the action of constant force in non-static medium with exponential scale factor by sampling over $10^{4}$ realizations. The running time PDF of Lévy walk behaves as Pareto distribution $\phi(\tau)=\frac{1}{\tau_{0}} \frac{\alpha}{\left(1+\tau / \tau_{0}\right)^{1+\alpha}}$ with $\tau_{0}=1$. For (a) and (d), we take $\mathrm{H}=0.1, \mathrm{H}=0.003$, respectively. The values of $\alpha$ respectively are $\alpha=0.5$ (square) and $\alpha=1.5$ (stars). For (b) and (e), we take $\mathrm{H}=-0.003$ and $\alpha=0.5$; and for (c) and (f), we take $\mathrm{H}=-0.003$ and $\alpha=1.5$

Here, we derive the concrete expression of $C$ and detailedly present it in (77). Correspondingly, the long-time asymptotic behaviour of the first two moments of Lévy walk moving under the combined action of constant force and exponential expansion medium in physical coordinate can be figured out, which respectively are

$$
\langle y(t)\rangle \sim \frac{a \alpha e^{H t}\left(-1+\alpha+H \tau_{0}\right)-a \alpha e^{H\left(t+\tau_{0}\right)}\left((-1+\alpha) \alpha+2 \alpha H \tau_{0}+H^{2} \tau_{0}^{2}\right) E_{1+\alpha}\left(H \tau_{0}\right)}{2 H^{2}\left(-1+\alpha e^{H \tau_{0}} \tau_{0}^{\alpha} H^{\alpha} \Gamma\left(-\alpha, H \tau_{0}\right)\right)}
$$

and

$$
\left\langle y^{2}(t)\right\rangle \sim C e^{2 H t} .
$$

However, the long-time asymptotic expressions of the first two moments in comoving coordinate rely on the category of $\alpha$ for $H<0$, respectively, behaving as

$$
\langle x(t)\rangle \sim \begin{cases}\frac{a}{4}(\alpha-2)(\alpha-1) e^{-H t} t^{2}, & \text { if } 0<\alpha<1, \\ \frac{a(\alpha-1)}{2(3-\alpha)} \tau_{0}^{\alpha-1} e^{-H t} t^{3-\alpha}, & \text { if } 1<\alpha<2\end{cases}
$$

and

$$
\left\langle x^{2}(t)\right\rangle \sim \begin{cases}\frac{a^{2}}{96}(\alpha-4)(\alpha-3)(\alpha-2)(\alpha-1) e^{-2 H t} t^{4}, & \text { if } 0<\alpha<1 \\ \frac{a^{2}(\alpha-1) \tau_{0}^{\alpha-1}}{4(5-\alpha)} e^{-2 H t} t^{5-\alpha}, & \text { if } 1<\alpha<2\end{cases}
$$

Again, we figure out the long-time asymptotic behavior of the first two moments in physical coordinates, respectively, being

$$
\langle y(t)\rangle \sim \begin{cases}\frac{a}{4}(\alpha-2)(\alpha-1) t^{2}, & \text { if } 0<\alpha<1 \\ \frac{a(\alpha-1)}{2(3-\alpha)} \tau_{0}^{\alpha-1} t^{3-\alpha}, & \text { if } 1<\alpha<2\end{cases}
$$



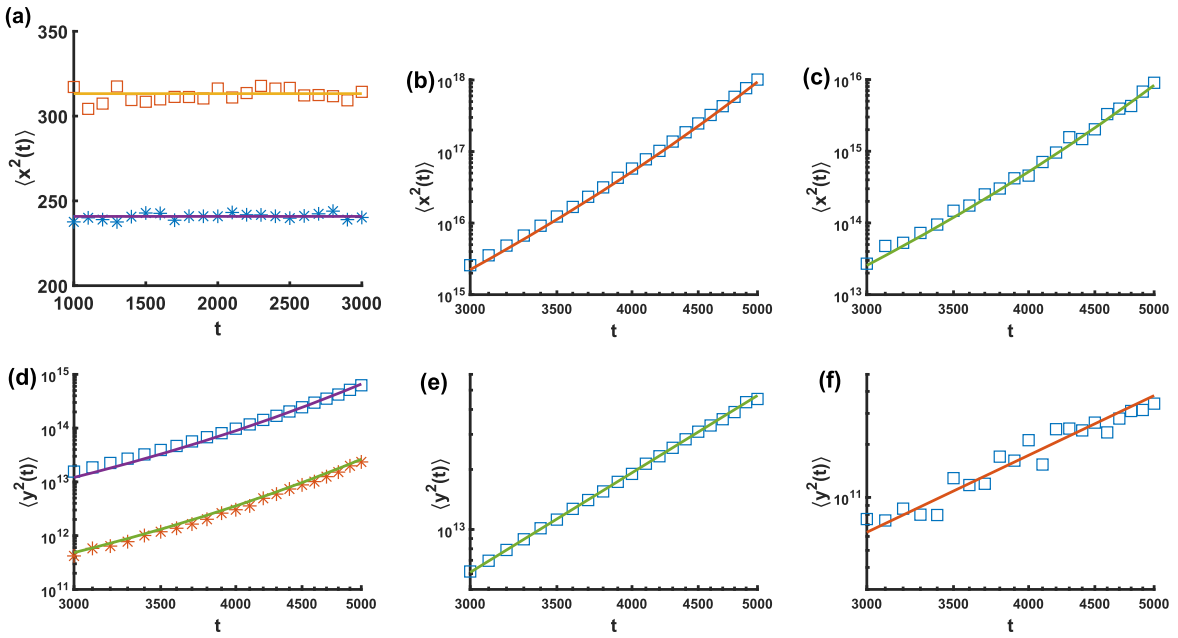

Fig. 10 Numerical simulations of MSDs of Lévy walk under the action of constant force in non-static medium with exponential scale factor by sampling over $10^{4}$ realizations. The walking time PDF of Lévy walk behaves as Pareto distribution $\phi(\tau)=\frac{1}{\tau_{0}} \frac{\alpha}{\left(1+\tau / \tau_{0}\right)^{1+\alpha}}$ with $\tau_{0}=1$. The other parameters are same with the ones in Fig. 9

and

$$
\left\langle y^{2}(t)\right\rangle \sim \begin{cases}\frac{a^{2}}{96}(\alpha-4)(\alpha-3)(\alpha-2)(\alpha-1) t^{4}, & \text { if } 0<\alpha<1, \\ \frac{a^{2}(\alpha-1) \tau_{0}^{\alpha-1}}{4(5-\alpha)} t^{5-\alpha}, & \text { if } 1<\alpha<2 .\end{cases}
$$

It should be noted that although the results in Eqs. (53) and (54) seem to be irrelevant to $H$, the exact forms do depend on $H$. For example, when $0<\alpha<1,\langle x(t)\rangle=\frac{a(1-\alpha) e^{-H t}}{4 H^{2}}(-2 \alpha+$ $\left.2 \alpha e^{H t}-2 \alpha H t+2 H^{2} t^{2}-\alpha H^{2} t^{2}\right)$. On the basis of Eq. (29), in physical coordinate we have $\langle y(t)\rangle=\frac{a(1-\alpha)}{4 H^{2}}\left(-2 \alpha+2 \alpha e^{H t}-2 \alpha H t+2 H^{2} t^{2}-\alpha H^{2} t^{2}\right)$.

When the non-static medium is described by exponential scale factor with Hubble constant $H>0$, Eqs. (49) and (50) tell us that the first two moments in physical coordinate grow unbounded for $t \rightarrow \infty$ in the way of $e^{H t}$ and $e^{2 H t}$ for $\alpha \in(0,1) \cup(1,2)$. Therefore, the dominative term of the overall diffusion process is the motion caused by the expansion of the medium. On the other hand, Eq. (48) shows that a stationary propagator will be involved in comoving coordinate for sufficiently long time for $\alpha \in(0,1) \cup(1,2)$ when the non-static medium is exponential expansion $H>0$. A superdiffusion can be found when the non-static medium is exponential contraction $H<0$. In this situation, the asymptotic behaviours of the first two moments rely on the category of $\alpha$. For example, the first two moments in physical coordinate respectively behave as $t^{2}$ and $t^{4}$ for $0<\alpha<1$ and appear as $t^{3-\alpha}$ and $t^{5-\alpha}$ for $1<\alpha<2$, which keep same with the first two moments of Lévy walk moving under the action of constant force in static medium. We come to a conclusion that the diffusion of the walker is mainly driven by constant external force when the medium is exponential contraction $H<0$. Furthermore, the average moments in both cases do not rely on the initial velocity of each step $v_{0}$. The above results are verified in Figs. 9 and 10. 


\subsubsection{Power-Law Scale Factor}

We calculate the long-time asymptotic behaviours of the first two moments in both coordinates when the scale factor of the non-static medium is power-law. As above, after some calculations we find that the asymptotic behaviours of the first two moments rely on the categories of $\alpha$. Specially, for $0<\alpha<1$, the first two moments in comoving coordinates respectively read

$$
\langle x(t)\rangle \sim \begin{cases}\frac{a(\alpha-1)(4+(\alpha-2) \beta) t_{0}^{\beta}}{4(\beta-2)} t^{2-\beta}, & \text { if } \beta<2, \\ \frac{a t_{0}^{2} \alpha}{2}(1-\alpha)(\gamma+\ln (t)), & \text { if } \beta=2, \\ C_{1}, & \text { if } \beta>2,\end{cases}
$$

and

$$
\left\langle x^{2}(t)\right\rangle \sim \begin{cases}\frac{a^{2}(1-\alpha)(8+(\alpha-4) \beta) t_{0}^{2 \beta}}{96(\beta-4)(\beta-3)(\beta-2)^{2}} t^{4-2 \beta}, & \text { if } \beta<2, \\ \frac{a^{2} \alpha^{2} t_{0}^{4}}{4}(\alpha-1)^{2}(\gamma+\ln (t))^{2}, & \text { if } \beta=2, \\ C_{2}, & \text { if } \beta>2 .\end{cases}
$$

Correspondingly, the first two moments in physical coordinates satisfy

$$
\langle y(t)\rangle \sim \begin{cases}\frac{a(\alpha-1)(4+(\alpha-2) \beta)}{4(\beta-2)} t^{2}, & \text { if } \beta<2 \\ \frac{a \alpha}{2}(\gamma+\ln (t)) t^{2}, & \text { if } \beta=2 \\ C_{1} t^{\beta}, & \text { if } \beta>2\end{cases}
$$

and

$$
\left\langle y^{2}(t)\right\rangle \sim \begin{cases}\frac{a^{2}(1-\alpha)(8+(\alpha-4) \beta)}{96(\beta-4)(\beta-3)(\beta-2)^{2}} t^{4}, & \text { if } \beta<2 \\ \frac{a^{2} \alpha^{2}}{4}(\alpha-1)^{2}(\gamma+\ln (t))^{2} t^{4}, & \text { if } \beta=2 \\ C_{2} t^{2 \beta}, & \text { if } \beta>2\end{cases}
$$

These results are verified in Figs. 11 and 12 .

On the other hand, for $1<\alpha<2$, the first two moments in comoving coordinates approximate to the following expressions for long time limit,

$$
\langle x(t)\rangle \sim \begin{cases}\frac{a(\alpha-1)(6-2 \alpha-2 \beta+\alpha \beta)}{2(\alpha-2)(\alpha-3)(3-\alpha-\beta)} t_{0}^{\beta} \tau_{0}^{\alpha-1} t^{3-\alpha-\beta}, & \text { if } \beta<3-\alpha, \\ \frac{a \alpha(\alpha-1)}{2(2-\alpha)} t_{0}^{3-\alpha} \tau_{0}^{\alpha-1}(\gamma+\ln (t)), & \text { if } \beta=3-\alpha, \\ C_{3}, & \text { if } \beta>3-\alpha,\end{cases}
$$

and

$$
\left\langle x^{2}(t)\right\rangle \sim \begin{cases}\frac{a^{2}(\alpha-1)(10+\alpha(\beta-2)-4 \beta) t_{0}^{2 \beta} \tau_{0}^{\alpha-1}}{2(\alpha-5)(\alpha-4)(5-\alpha-2 \beta)} t^{5-\alpha-2 \beta}, & \text { if } \beta<\frac{5-\alpha}{2} \\ \frac{a^{2} \alpha(\alpha-1) t_{0}^{5-\alpha} \tau_{0}^{\alpha-1}}{4(4-\alpha)}(\gamma+\ln (t)), & \text { if } \beta=\frac{5-\alpha}{2} \\ C_{4}, & \text { if } \beta>\frac{5-\alpha}{2}\end{cases}
$$

Similarly, in physical coordinate, we have

$$
\langle y(t)\rangle \sim \begin{cases}\frac{a(\alpha-1)(6-2 \alpha-2 \beta+\alpha \beta)}{2(\alpha-2)(\alpha-3)(3-\alpha-\beta)} \tau_{0}^{\alpha-1} t^{3-\alpha}, & \text { if } \beta<3-\alpha, \\ \frac{a \alpha(\alpha-1)}{2(2-\alpha)} \tau_{0}^{\alpha-1} t^{3-\alpha}(\gamma+\ln (t)) & \text { if } \beta=3-\alpha, \\ C_{3} t^{\beta}, & \text { if } \beta>3-\alpha\end{cases}
$$



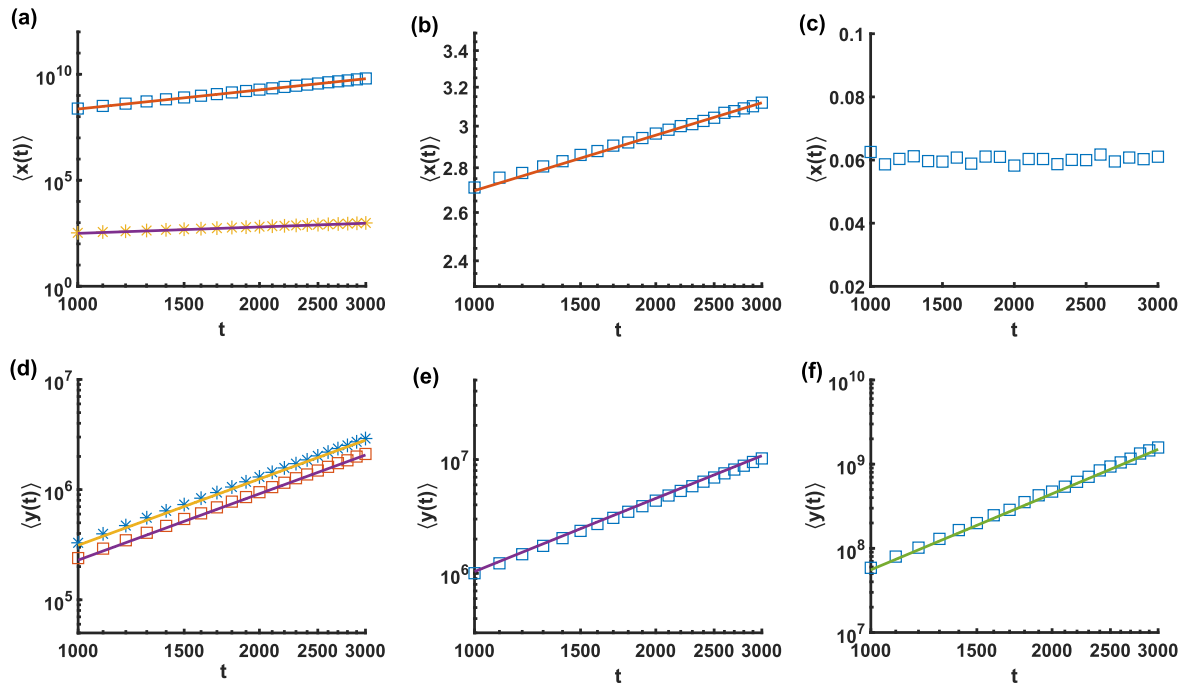

Fig. 11 Numerical simulations of the average moments of Lévy walk under the action of constant force in non-static medium with power-law scale factor by sampling over $10^{4}$ realizations. The running time PDF of Lévy walk behaves as Pareto distribution $\phi(\tau)=\frac{1}{\tau_{0}} \frac{\alpha}{\left(1+\tau / \tau_{0}\right)^{1+\alpha}}$ with $\tau_{0}=1$ and $\alpha=0.5$. Here, we assume $t_{0}=1$. For (a) and (d), we take $\beta=-1$ (squares) and $\beta=1$ (stars). For (b) and (e), we take $\beta=2$ and for (c) and (f), $\beta=3$
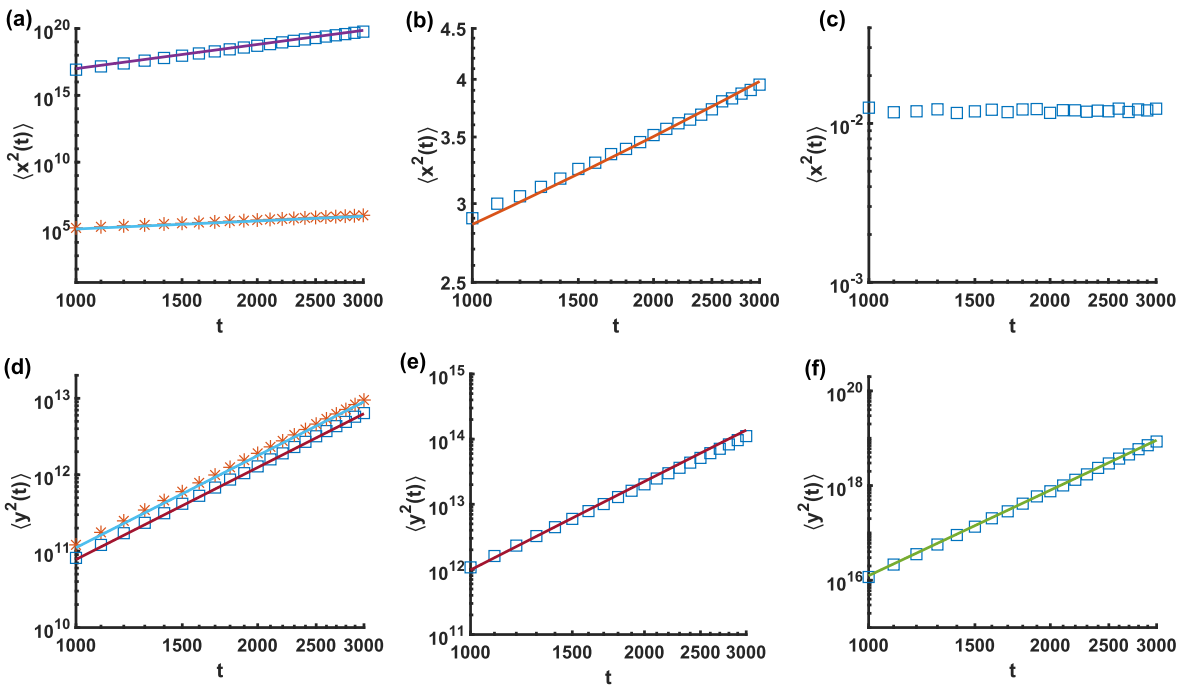

Fig. 12 Numerical simulations of MSDs of Lévy walk under the action of constant force in non-static medium with power-law scale factor by sampling over $10^{4}$ realizations. The running time PDF of Lévy walk behaves as Pareto distribution $\phi(\tau)=\frac{1}{\tau_{0}} \frac{\alpha}{\left(1+\tau / \tau_{0}\right)^{1+\alpha}}$ with $\tau_{0}=1$ and $\alpha=0.5$. The other parameters are same with the ones in Fig. 11 

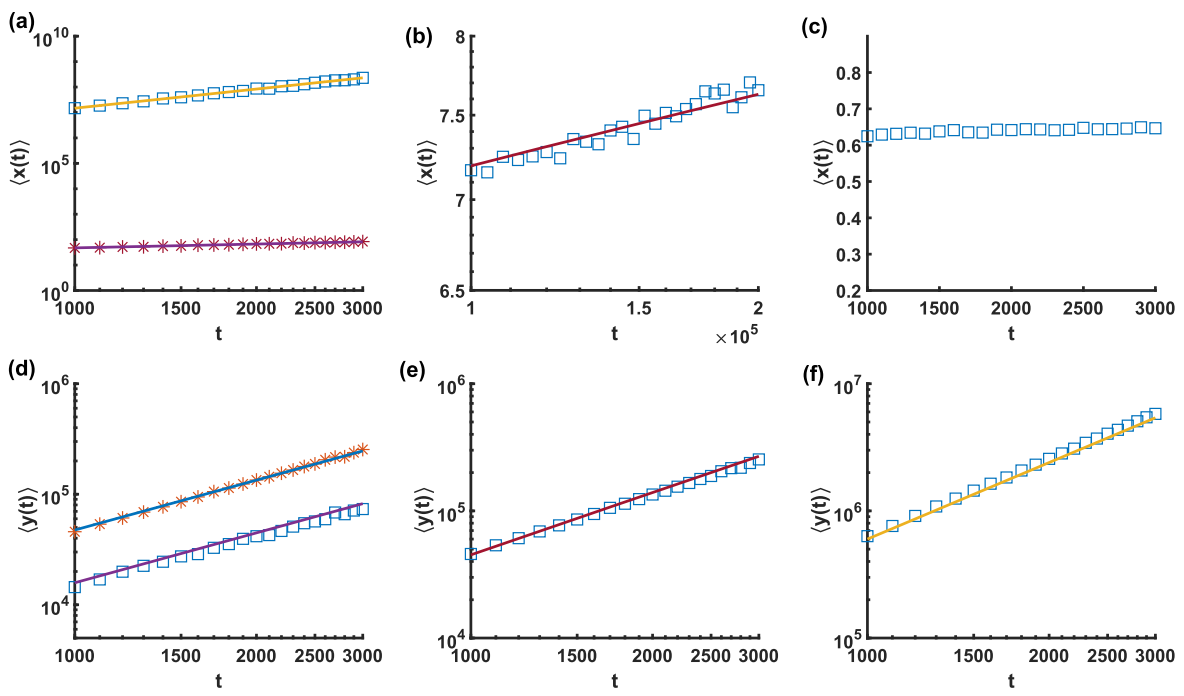

Fig. 13 Numerical simulations of the average moments of Lévy walk under the action of constant force in non-static medium with power-law scale factor by sampling over $10^{4}$ realizations. The running time PDF of Lévy walk behaves as Pareto distribution $\phi(\tau)=\frac{1}{\tau_{0}} \frac{\alpha}{\left(1+\tau / \tau_{0}\right)^{1+\alpha}}$ with $\tau_{0}=1$ and $\alpha=1.5$. Here, we assume $t_{0}=1$. For (a) and (d), we take $\beta=-1$ (squares) and $\beta=1$ (stars). For (b) and (e), we take $\beta=1.5$ and for (c) and (f), $\beta=2$

and

$$
\left\langle y^{2}(t)\right\rangle \sim \begin{cases}\frac{a^{2}(\alpha-1)(10+\alpha(\beta-2)-4 \beta) \tau_{0}^{\alpha-1}}{2(\alpha-5)(\alpha-4)(5-\alpha-2 \beta)} t^{5-\alpha}, & \text { if } \beta<\frac{5-\alpha}{2}, \\ \frac{a^{2} \alpha(\alpha-1) \tau_{0}^{\alpha-1}}{4(4-\alpha)}(\gamma+\ln (t)) t^{5-\alpha}, & \text { if } \beta=\frac{5-\alpha}{2}, \\ C_{4} t^{2 \beta}, & \text { if } \beta>\frac{5-\alpha}{2} .\end{cases}
$$

We verify the above results in Figs. 13 and 14. Compared with the traditional Lévy walk model in static medium, we conclude that the combined action of constant force and nonstatic medium accelerates the motion of the diffusion particles. Notably, the dominative term of the diffusion process depends on the range of $\alpha$ and $\beta$. For example, if $0<\alpha<1$, the first two moments in physical coordinate respectively appear as $t^{2}$ and $t^{4}$ when $\beta<2$, which are same with the behaviours of particles in static medium under the action of constant potential. Furthermore, we prove that the non-static medium hardly affects the motion of the walker when the external potential comes into play. The dominative term of the diffusion process is the motion caused by external potential. However, the MSD in physical coordinate behaves as $t^{2 \beta}$ for sufficiently long time when $\beta>2$, which are similar to the result of MSD of pure Lévy walk model moving in non-static medium with power-law scale factor [75]. Accordingly, we state that the diffusion process is mainly driven by the non-static expansion. Following the same way, for $1<\alpha<2$, we conclude the displacement of the particles is mainly driven by external potential when $\beta<\frac{5-\alpha}{2}$, whereas it is mainly driven by the non-static medium when $\beta>\frac{5-\alpha}{2}$. 

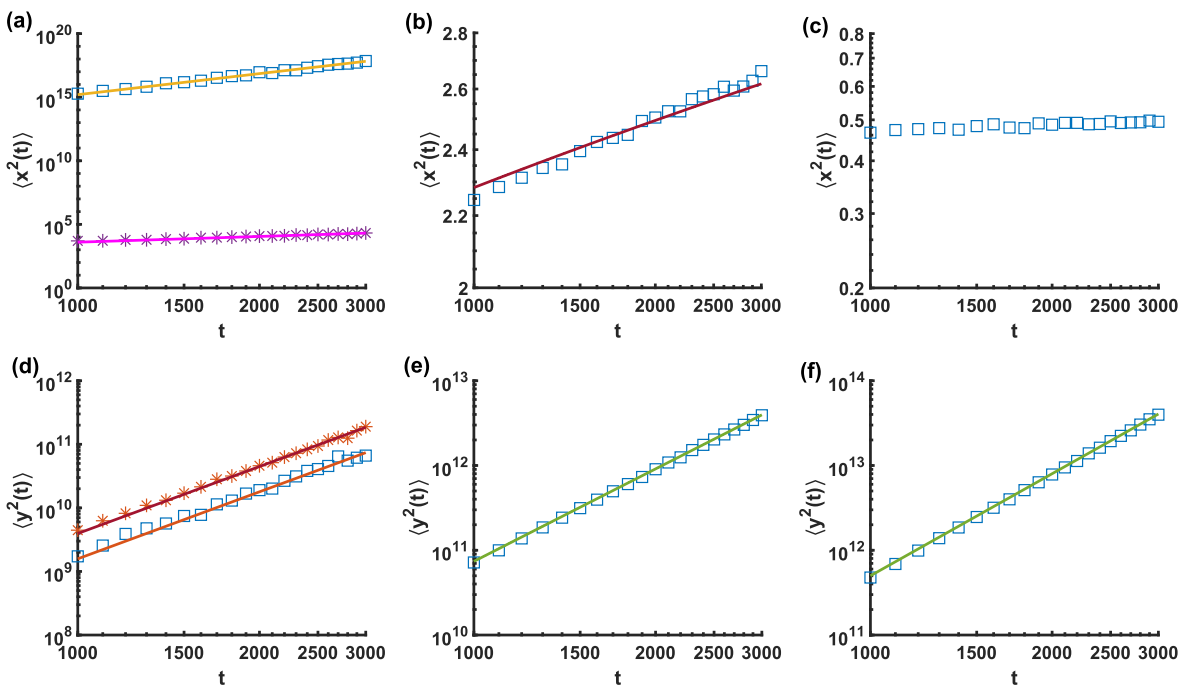

Fig. 14 Numerical simulations of MSDs of Lévy walk under the action of constant force in non-static medium with power-law scale factor by sampling over $10^{4}$ realizations. The running time PDF of Lévy walk behaves as Pareto distribution $\phi(\tau)=\frac{1}{\tau_{0}} \frac{\alpha}{\left(1+\tau / \tau_{0}\right)^{1+\alpha}}$ with $\tau_{0}=1$ and $\alpha=1.5$. Expect that in (b) and (e), we take $\beta=1.75$. The other parameters in (a) and (d) as well as in (c) and (f) keep same with the ones of Fig. 13

\section{Conclusion}

In this paper we establish a Lévy walk model moving under the action of a constant external force in one dimensional non-static medium and study the dynamics of the model. First, we build a general equation, governing the PDF of the position of the particles in comoving coordinate by involving the scale factor. Next, we use Hermite orthogonal polynomials to approach the solution of the equation and some representative statistics are obtained by a series of functions to be determined. Furthermore, combining with some representative examples of the running time PDFs $\phi(t)$ and scale factors $d(t)$, we get the statistical properties in both coordinates through analytical analyses and numerical simulations.

For Lévy walk with the running time of exponential distribution, the motion of the particle is mainly driven by the non-static medium when the scale factor is an exponential function. Specially, for exponential expansion medium $H>0$, the first two moments in comoving coordinate tend to be a constant, resulting in growing exponentially in physical coordinate. However, for exponential contraction medium $H<0$, the first two moments in comoving and physical coordinates respectively are exponential growth and constant for sufficiently long time. Thus, a stationary propagator function emerges for the process with $H>0$ in comoving coordinate or $H<0$ in physical coordinate. We analyze the stationary distribution by numerical simulations. Next, we calculate the kurtosis of stationary distribution in physical coordinate for $H<0$, which reveals that $K$ relies on $v_{0}, a, H, \lambda$. For any fixed $H, v_{0}, a$, we conclude that the stationary PDF is leptokurtic for small $\lambda$ and is Gaussian for large $\lambda$. The situation is completely different for non-static medium with power-law scale factor. 
The first two moments in physical coordinate respectively behave as $t$ and $t^{2}$ after sufficiently long time when $\beta<1$, and are consistent with the first two moments of Lévy walk in static medium in constant force field. Therefore, the dominative term of the diffusion process is the motion caused by constant external force. On the other hand, the motion is determined by the non-static medium when $\beta>1$, since in non-static medium the exponent of the MSD in physical coordinate is same with the exponent of the MSD of pure Lévy walk. Besides, the initial value of velocity $v_{0}$ in each step has no influence on the average displacement in both scale factors $d(t)$.

For Lévy walk with Pareto distributed running time, we also analyze the long time dynamic behaviours of Lévy walk under the combined action of constant force and the non-static medium. The first two moments in physical coordinate exponentially grow when the scale factor of the non-static medium exponentially expands with $H>0$, i.e., the expansion of the medium dominates the overall process. The long-time asymptotic behaviour of the first two moments relies on the category of $\alpha$ when the medium exponentially contracts with $H<0$, both of which are same with the first two moments of Lévy walk in constant force field in static medium for $\alpha \in(0,1) \cup(1,2)$. We conclude that the constant force drives the overall diffusion process. Finally, after sufficiently long time, we analyze the first two moments in physical coordinate when the non-static medium is described by power law scale factor. A critical value $\beta_{c}$ can be found in this case. When $\beta<\beta_{c}$ the MSDs in physical coordinate are same with the MSDs of Lévy walk in constant force field in static medium for $\alpha \in(0,1) \cup(1,2)$, i.e., the dominative term of the process is the motion induced by external constant potential. However, the MSDs in physical coordinate are similar to the results of MSDs of pure Lévy walk model moving in non-static medium when $\beta>\beta_{c}$, which implies that the non-static medium dominates the overall diffusion process. In addition, the critical value of $\beta_{c}$ depends on the categories of $\alpha$; if $\alpha \in(0,1)$ then $\beta_{c}=2$ while if $\alpha \in(1,2)$ then $\beta_{c}=(5-\alpha) / 2$.

Acknowledgements This work was supported by the National Natural Science Foundation of China under Grant No. 12071195, and the AI and Big Data Funds under Grant No. 2019620005000775.

\section{Declarations}

Data availability All data and models generated or used during the study appear in this article.

\section{A: A Brief Introduction of Hermite Polynomials}

Hermite polynomials are a set of orthogonal polynomials defined on $(-\infty, \infty)$ with weight function $e^{-x^{2}}$ [3]. The standardized Hermite polynomial is given as

$$
H_{n}(x)=(-1)^{n} e^{x^{2}} \frac{d^{n}}{d x^{n}} e^{-x^{2}}
$$

Furthermore, its orthogonality can be represented as

$$
\int_{-\infty}^{\infty} H_{n}(x) H_{m}(x) e^{-x^{2}} d x=\sqrt{\pi} 2^{n} n ! \delta_{n, m},
$$


where $\delta_{n, m}$ is the Kronecker delta function. By Taylor's expansion, it has

$$
H_{n}(x+y)=\sum_{k=0}^{n}\left(\begin{array}{l}
n \\
k
\end{array}\right) H_{k}(y)(2 x)^{n-k},
$$

and the following holds

$$
H_{n}(\gamma x)=\sum_{j=0}^{\left\lfloor\frac{n}{2}\right\rfloor} \gamma^{n-2 j}\left(\gamma^{2}-1\right)^{j}\left(\begin{array}{c}
n \\
2 j
\end{array}\right) \frac{(2 j) !}{j !} H_{n-2 j}(x),
$$

where $\left\lfloor\frac{n}{2}\right\rfloor$ is the biggest integer smaller than $\frac{n}{2}$. The special value of Hermite polynomials is the value evaluated at zero argument $H_{n}(0)$, which are called Hermite number,

$$
H_{n}(0)= \begin{cases}0, & \text { if } n \text { is odd, } \\ (-1)^{\frac{n}{2}} 2^{\frac{n}{2}}(n-1) ! !, & \text { if } n \text { is even. }\end{cases}
$$

In particular,

$$
H_{0}(x)=1, \quad H_{1}(x)=2 x, \quad H_{2}(x)=4 x^{2}-2 .
$$

\section{B: Derivations of (16) and (17)}

Plugging (14) into (12) yields

$$
\begin{aligned}
\sum_{n=0}^{\infty} H_{n}(x) T_{n}(t) e^{-x^{2}=} & \frac{1}{2} \int_{0}^{t} \sum_{n=0}^{\infty} H_{n}\left(x-\frac{\frac{1}{2} a \tau^{2}+v_{0} \tau}{d(t)}\right) T_{n}(t-\tau) \\
& \exp \left[-\left(x-\frac{\frac{1}{2} a \tau^{2}+v_{0} \tau}{d(t)}\right)^{2}\right] \phi(\tau) d \tau \\
& +\frac{1}{2} \int_{0}^{t} \sum_{n=0}^{\infty} H_{n}\left(x-\frac{\frac{1}{2} a \tau^{2}-v_{0} \tau}{d(t)}\right) T_{n}(t-\tau) \\
& \exp \left[-\left(x-\frac{\frac{1}{2} a \tau^{2}-v_{0} \tau}{d(t)}\right)^{2}\right] \phi(\tau) d \tau+P_{0}(x) \delta(t) .
\end{aligned}
$$

Multiply both sides of (69) by $H_{m}(x), m=0,1,2, \cdots$, and integrate $x$ over $(-\infty,+\infty)$. In accordance with the orthogonality of Hermite polynomials (64), the left hand of (69) becomes

$$
\int_{-\infty}^{+\infty} \sum_{n=0}^{\infty} H_{n}(x) T_{n}(t) H_{m}(x) e^{-x^{2}} d x=\sqrt{\pi} 2^{m} m ! T_{m}(t) .
$$

We next focus on the first term of the right hands of (69). After the above manipulations, it can be written as

$$
\begin{gathered}
\frac{1}{2} \int_{0}^{t} \phi(\tau) T_{n}(t-\tau) \int_{-\infty}^{+\infty} H_{m}(x) \sum_{n=0}^{\infty} H_{n}\left(x-\frac{\frac{1}{2} a \tau^{2}+v_{0} \tau}{d(t)}\right) \\
\exp \left[-\left(x-\frac{\frac{1}{2} a \tau^{2}+v_{0} \tau}{d(t)}\right)^{2}\right] d x d \tau
\end{gathered}
$$




$$
\begin{aligned}
& =\frac{1}{2} \int_{0}^{t} \phi(\tau) T_{n}(t-\tau) \int_{-\infty}^{+\infty} H_{m}\left(y+\frac{\frac{1}{2} a \tau^{2}+v_{0} \tau}{d(t)}\right) \sum_{n=0}^{\infty} H_{n}(y) \exp \left(-y^{2}\right) d y d \tau \\
& =\frac{1}{2} \int_{0}^{t} \phi(\tau) T_{n}(t-\tau) \int_{-\infty}^{+\infty} \sum_{k=0}^{m}\left(\begin{array}{c}
m \\
k
\end{array}\right) H_{k}(y)\left(\frac{a \tau^{2}+2 v_{0} \tau}{d(t)}\right)^{m-k} \sum_{n=0}^{\infty} H_{n}(y) \exp \left(-y^{2}\right) d y d \tau \\
& =\frac{1}{2} \sum_{k=0}^{m}\left(\begin{array}{c}
m \\
k
\end{array}\right) \int_{0}^{t} \phi(\tau) T_{n}(t-\tau)\left(\frac{a \tau^{2}+2 v_{0} \tau}{d(t)}\right)^{m-k} \int_{-\infty}^{+\infty} H_{k}(y) \sum_{n=0}^{\infty} H_{n}(y) \exp \left(-y^{2}\right) d y d \tau \\
& =\frac{1}{2} \sum_{k=0}^{m} \frac{m !}{k !(m-k) !} \int_{0}^{t} \sqrt{\pi} 2^{k} k !\left(\frac{a \tau^{2}+2 v_{0} \tau}{d(t)}\right)^{m-k} T_{k}(t-\tau) \phi(\tau) d \tau \\
& =\frac{1}{2} \sum_{k=0}^{m} \frac{m ! \sqrt{\pi} 2^{k}}{(m-k) !} \int_{0}^{t}\left(\frac{a \tau^{2}+2 v_{0} \tau}{d(t)}\right)^{m-k} T_{k}(t-\tau) \phi(\tau) d \tau .
\end{aligned}
$$

The above procedures are using the properties of Hermite polynomials presented in (64) and (65) in Appendix A. By the same procedure, the second term of the right hand side of (69) can be simplified as $\frac{1}{2} \sum_{k=0}^{m} \frac{m ! \sqrt{\pi} 2^{k}}{(m-k) !} \int_{0}^{t}\left(\frac{a \tau^{2}-2 v_{0} \tau}{d(t)}\right)^{m-k} T_{k}(t-\tau) \phi(\tau) d \tau$. Considering that the initial distribution of particles behaves as Dirac-delta function, i.e., $P_{0}(x)=\delta(x)$, the third term is reduced to $\int_{-\infty}^{+\infty} H_{m}(x) \delta(x) \delta(t) d x=\delta(t) H_{m}(0)$. Then we reach the iteration relation

$$
\begin{aligned}
\sqrt{\pi} 2^{m} m ! T_{m}(t)= & \frac{1}{2} \sum_{k=0}^{m} \frac{m ! \sqrt{\pi} 2^{k}}{(m-k) !} \int_{0}^{t}\left[\left(\frac{a \tau^{2}+2 v_{0} \tau}{d(t)}\right)^{m-k}+\left(\frac{a \tau^{2}-2 v_{0} \tau}{d(t)}\right)^{m-k}\right] \\
& \times T_{k}(t-\tau) \phi(\tau) d \tau+\delta(t) H_{m}(0) .
\end{aligned}
$$

Similarly, by substituting (15) into (13), the relation between $R_{m}(t)$ and $T_{m}(t)$ can be obtained as

$$
\begin{aligned}
\sqrt{\pi} 2^{m} m ! R_{m}(t)= & \frac{1}{2} \sum_{k=0}^{m} \frac{m ! \sqrt{\pi} 2^{k}}{(m-k) !} \int_{0}^{t}\left[\left(\frac{a \tau^{2}+2 v_{0} \tau}{d(t)}\right)^{m-k}+\left(\frac{a \tau^{2}-2 v_{0} \tau}{d(t)}\right)^{m-k}\right] \\
& T_{k}(t-\tau) \psi(\tau) d \tau .
\end{aligned}
$$

With the help of convolution theorem, Eqs. (15) and (16) can be obtained by taking Laplace transform on (72) and (73).

\section{C: Some Specific Values}

The value of Kurtosis of Lévy walk under the action of external constant potential in non-static medium with exponential contraction scale factor reads

$$
\begin{aligned}
K & \sim \frac{1}{\left(5 a^{2} H^{3}-11 a^{2} H^{2} \lambda+6 a^{2} H \lambda^{2}-a^{2} \lambda^{3}+2 H^{3} \lambda^{2} v_{0}^{2}-5 H^{2} \lambda^{3} v_{0}^{2}+4 H \lambda^{4} v_{0}^{2}-\lambda^{5} v_{0}^{2}\right)^{2}} \\
& \times\left[\frac{3 a^{4}\left(105264 H^{14}-923232 H^{13} \lambda+3664808 H^{12} \lambda^{2}-8701896 H^{11} \lambda^{3}+13759459 H^{10} \lambda^{4}\right)}{(H-\lambda)^{2}(2 H-\lambda)^{4}(3 H-\lambda)^{2}}\right. \\
& +\frac{3 a^{4}\left(-15255944 H^{9} \lambda^{5}+12151591 H^{8} \lambda^{6}-7007718 H^{7} \lambda^{7}+2915137 H^{6} \lambda^{8}-862504 H^{5} \lambda^{9}\right)}{(H-\lambda)^{2}(2 H-\lambda)^{4}(3 H-\lambda)^{2}} \\
& +\frac{3 a^{4}\left(176724 H^{4} \lambda^{10}-23936 H^{3} \lambda^{11}+1968 H^{2} \lambda^{12}-82 H \lambda^{13}+\lambda^{14}\right)}{(H-\lambda)^{2}(2 H-\lambda)^{4}(3 H-\lambda)^{2}}
\end{aligned}
$$




$$
\begin{aligned}
& +\frac{6 a^{2} \lambda^{2}\left(22752 H^{12}-154272 H^{11} \lambda+462896 H^{10} \lambda^{2}-809024 H^{9} \lambda^{3}+911540 H^{8} \lambda^{4}\right) v_{0}^{2}}{(3 H-\lambda)^{2}(2 H-\lambda)^{4}} \\
& +\frac{6 a^{2} \lambda^{2}\left(-694298 H^{7} \lambda^{5}+365279 H^{6} \lambda^{6}-133283 H^{5} \lambda^{7}+33349 H^{4} \lambda^{8}-5565 H^{3} \lambda^{9}\right) v_{0}^{2}}{(3 H-\lambda)^{2}(2 H-\lambda)^{4}} \\
& +\frac{6 a^{2} \lambda^{2}\left(589 H^{2} \lambda^{10}-36 H \lambda^{11}+\lambda^{12}\right) v_{0}^{2}}{(3 H-\lambda)^{2}(2 H-\lambda)^{4}} \\
& \left.+\frac{3(H-\lambda)^{4}(4 H-\lambda) \lambda^{4}\left(8 H^{3}-8 H^{2} \lambda+2 H \lambda^{2}-\lambda^{3}\right) v_{0}^{4}}{(2 H-\lambda)^{2}}\right] .
\end{aligned}
$$

Further, we consider $K$ as a function of $v_{0}$ for any fixed $H, \lambda, a$; after sufficiently long time, for small $v_{0}$, there is

$$
\begin{aligned}
K_{v_{0} \rightarrow 0} \sim & \frac{3\left(105264 H^{14}-923232 H^{13} \lambda+3664808 H^{12} \lambda^{2}-8701896 H^{11} \lambda^{3}+13759459 H^{10} \lambda^{4}\right)}{(H-\lambda)^{2}(2 H-\lambda)^{4}(3 H-\lambda)^{2}\left(5 H^{3}-11 H^{2} \lambda+6 H \lambda^{2}-\lambda^{3}\right)^{2}} \\
& +\frac{3\left(-15255944 H^{9} \lambda^{5}+12151591 H^{8} \lambda^{6}-7007718 H^{7} \lambda^{7}+2915137 H^{6} \lambda^{8}\right)}{(H-\lambda)^{2}(2 H-\lambda)^{4}(3 H-\lambda)^{2}\left(5 H^{3}-11 H^{2} \lambda+6 H \lambda^{2}-\lambda^{3}\right)^{2}} \\
& +\frac{3\left(-862504 H^{5} \lambda^{9}+176724 H^{4} \lambda^{10}-23936 H^{3} \lambda^{11}+1968 H^{2} \lambda^{12}-82 H \lambda^{13}+\lambda^{14}\right)}{(H-\lambda)^{2}(2 H-\lambda)^{4}(3 H-\lambda)^{2}\left(5 H^{3}-11 H^{2} \lambda+6 H \lambda^{2}-\lambda^{3}\right)^{2}} ;
\end{aligned}
$$

similarly, for large $v_{0}$, it has

$$
K_{v_{0} \rightarrow \infty} \sim \frac{3\left(32 H^{4}-40 H^{3} \lambda+16 H^{2} \lambda^{2}-6 H \lambda^{3}+\lambda^{4}\right)}{(2 H-\lambda)^{4}} .
$$

If we consider $K$ as a function of $a$ for any fixed $H, \lambda, v_{0}$. By the same method, we find that $K_{a \rightarrow 0}=K_{v_{0} \rightarrow \infty}$ and $K_{a \rightarrow \infty}=K_{v_{0} \rightarrow 0}$.

The value of $C$ in (48) is

$$
\begin{aligned}
C= & \frac{\alpha a^{2}}{64 H^{4}\left(-1+\alpha e^{H \tau_{0}} H^{\alpha} \tau_{0}^{\alpha} \Gamma\left(-\alpha, H \tau_{0}\right)\right)\left(-1+2^{\alpha} \alpha e^{2 H \tau_{0}} H^{\alpha} \tau_{0}^{\alpha} \Gamma\left(-\alpha, 2 H \tau_{0}\right)\right)} \\
& \times\left[6+\alpha(-3+\alpha(-10+7 \alpha))-4 H \tau_{0}+2 \alpha(-5+9 \alpha) H \tau_{0}+4(1+\alpha) H^{2} \tau_{0}^{2}-8 H^{3} \tau_{0}^{3}\right. \\
& -16 H^{2}(-1+\alpha) v_{0}^{2}-32 H^{3} \tau_{0} v_{0}^{2}-26 H e^{H \tau_{0}} E_{1+\alpha}\left(H \tau_{0}\right) a^{2} \alpha^{3} \tau_{0}+16 H^{4} \tau_{0}^{4} a^{2} e^{2 H \tau_{0}} \\
& +a^{2} e^{2 H \tau_{0}}\left((1-\alpha) \alpha(-6+\alpha(-3+7 \alpha))+16 \alpha\left(1+\alpha-2 \alpha^{2}\right) H \tau_{0}+8(1-5 \alpha) \alpha H^{2} \tau_{0}^{2}\right) \\
& +e^{2 H \tau_{0}} 16 H^{2}\left((-1+\alpha) \alpha+4 \alpha H \tau_{0}+4 H^{2} \tau_{0}^{2}\right) v_{0}^{2} E_{1+\alpha}\left(2 H \tau_{0}\right) \\
& +\alpha e^{H \tau_{0}} E_{1+\alpha}\left(H \tau_{0}\right) a^{2}\left(\left(-6-7 \alpha^{3}+10 \alpha^{2}\right)+\alpha\left(3+2 H \tau_{0}\left(9-14 H \tau_{0}\right)\right)\right) \\
& +\alpha e^{H \tau_{0}} E_{1+\alpha}\left(H \tau_{0}\right) a^{2} 4 H \tau_{0}\left(1+H \tau_{0}\left(1-2 H \tau_{0}\right)\right)+16 \alpha e^{H \tau_{0}} E_{1+\alpha}\left(H \tau_{0}\right) H^{2}(-1+\alpha) v_{0}^{2} \\
& +e^{2 H \tau_{0}} a^{2}\left((-1+\alpha) \alpha(-6+\alpha(-3+7 \alpha))+8(-1+\alpha) \alpha(2+5 \alpha) H \tau_{0}\right) E_{1+\alpha}\left(2 H \tau_{0}\right) \\
& +e^{2 H \tau_{0}} a^{2} 16 \alpha(-1+5 \alpha) H^{2} \tau_{0}^{2} E_{1+\alpha}\left(2 H \tau_{0}\right)+32 \alpha H^{3} \tau_{0} v_{0}^{2} e^{H \tau_{0}} E_{1+\alpha}\left(H \tau_{0}\right) \\
& +64 a^{2} \alpha H^{3} \tau_{0}^{3} e^{2 H \tau_{0}} E_{1+\alpha}\left(2 H \tau_{0}\right)+e^{2 H \tau_{0}} 16 H^{4} a^{2} \tau_{0}^{4} E_{1+\alpha}\left(2 H \tau_{0}\right) \\
& \left.-e^{2 H \tau_{0}} 16 H^{2}\left((-1+\alpha) \alpha+4 \alpha H \tau_{0}+4 H^{2} \tau_{0}^{2}\right) v_{0}^{2} E_{1+\alpha}\left(2 H \tau_{0}\right)\right] .
\end{aligned}
$$




\section{References}

1. Aloisio, R., Berezinsky, V., Gazizov, A.Z.: The problem of superluminal diffusion of relativistic particles and its phenomenological solution. Astrophys. J. 693, 1275 (2009)

2. Averbukh, I., Ben-Zvi, D., Mishra, S., Barkai, N.: Scaling morphogen gradients during tissue growth by a cell division rule. Development 141, 2150-2156 (2014)

3. Abramowitz, M., Stegun, I.A.: Handbook of Mathematical Functions with Formulas, Graphs, and Mathematical Tables. United States Department of Commerce, Washington (1972)

4. Brown, R.: Mikroskopische beobachtungen über die im pollen der pflanzen enthaltenen partikeln, und über das allgemeine vorkommen activer molecüle in organischen und unorganischen körpern. Ann. Phys. Chem. 14, 294-313 (1828)

5. Bouchaud, J.-P., Georges, A.: Anomalous diffusion in disordered media: statistical mechanisms, models and physical applications. Phys. Rep. 195, 127-293 (1990)

6. Boffetta, G., Sokolov, I.M.: Relative dispersion in fully developed turbulence: the Richardson's law and intermittency corrections. Phys. Rev. Lett. 88, 094501 (2002)

7. Barkai, E., Fleurov, V., Klafter, J.: One-dimensional stochastic Lévy-Lorentz gas. Phys. Rev. E 61, 1164 (2000)

8. Binder, B.J., Landman, K.A., Simpson, M.J., Mariani, M., Newgreen, D.F.: Modeling proliferative tissue growth: ageneral approach and an avian case study. Phys. Rev. E 78, 031912 (2008)

9. Berezinsky, V., Gazizov, A.Z.: Diffusion of cosmic rays in the expanding universe. I. Astrophys. J. 643, 8 (2006)

10. Berezinsky, V., Gazizov, A.Z.: Diffusion of cosmic rays in the expanding universe. II. Energy spectra of ultra-high energy cosmic rays. Astrophys. J. 669, 684 (2007)

11. Becker-Kern, P., Meerschaert, M.M., Scheffler, H.-P.: Limit theorems for coupled continuous time random walks. Ann. Probab. 32, 730-756 (2004)

12. Caspi, A., Granek, R., Elbaum, M.: Enhanced diffusion in active intracellular transport. Phys. Rev. Lett. 85, 5655 (2000)

13. Chen, K.J., Wang, B., Granick, S.: Memoryless self-reinforcing directionality in endosomal active transport within living cells. Nat. Mater. 14, 589-593 (2015)

14. Chechkin, A.V., Klafter, J., Gonchar, V.Y., Metzler, R., Tanatarov, L.V.: Bifurcation, bimodality, and finite variance in confined Lévy flights. Phys. Rev. E 67, 010102 (2003)

15. Chechkin, A.V., Gonchar, V.Y.: Linear relaxation processes governed by fractional symmetric kinetic equations. Journ. Eksper. Theor. Phys. 91, 635-651 (2000)

16. Coffey, W.T., Kalmykov, Y.P.: The Langevin Equation with Applications to Stochastic Problems in Physics, Chemistry, and Electrical Enginering. World Scientific, Singapore (2012)

17. Crampin, E.J., Hackborn, W.W., Maini, P.K.: Pattern formation in reaction-diffusion models with nonuniform domain growth. Bull. Math. Biol. 64, 747-769 (2002)

18. Crampin, E.J., Gaffney, E., Maini, P.: Reaction and diffusion on growing domains: scenarios for robust pattern formation. Bull. Math. Biol. 61, 1093-1120 (1999)

19. Crampin, E.J., Maini, P.K.: Modelling biological pattern formation: the role of domain growth. Comments Theor. Biol. 6, 229-249 (2001)

20. Dix, J.A., Verkman, A.S.: Crowding effects on diffusion in solutions and cells. Annu. Rev. Biophys. 37, 247-263 (2008)

21. Fogedby, H.C.: Langevin equations for continuous time Lévy flights. Phys. Rev. E 50, 1657 (1994)

22. Fogedby, H.C.: Lévy flights in random environments. Phys. Rev. Lett. 73, 2517 (1994)

23. Fioriti, V., Fratichini, F., Chiesa, S., Moriconi, C.: Lévy foraging in a dynamic environment-extending the Lévy search. Int. J. Adv. Robot. Syst. 12, 98 (2015)

24. Fogedby, H.C.: Lévy flights in quenched random force fields. Phys. Rev. E 58, 1690 (1998)

25. Friedrich, R., Jenko, F., Baule, A., Eule, S.: Anomalous diffusion of inertial, weakly damped particles. Phys. Rev. Lett. 96, 230601 (2006)

26. Friedrich, R., Jenko, F., Baule, A., Eule, S.: Exact solution of a generalized Kramers-Fokker-Planck equation retaining retardation effects. Phys. Rev. E 74, 041103 (2006)

27. Gross, B., Zheng, Z., Liu, S., Chen, X., Sela, A., Li, J., Li, D., Havlin, S.: Erratum: spatio-temporal propagation of COVID-19 pandemics. EPL 131, 58003 (2020)

28. Gillespie, D.T.: Simple Brownian Diffusion: An Introduction to the Standard Theoretical Model. Oxford University Press, Oxford (2012)

29. Gardiner, C.: Stochastic Methods: A Handbook for the Natural and Social Sciences. Springer, Berlin (2009)

30. Höfling, F., Franosch, T.: Anomalous transport in the crowded world of biological cells. Rep. Prog. Phys. 76, 046602 (2013) 
31. Haba, Z.: Einstein gravity of a diffusing fluid. Class. Quantum Grav. 31, 075011 (2014)

32. Jeon, J.-H., Leijnse, N., Oddershede, L.B., Metzler, R.: Anomalous diffusion and power-law relaxation of the time averaged mean squared displacement in worm-like micellar solutions. New J. Phys. 15, 045011 (2013)

33. Jeon, J.-H., Monne, H.M.-S., Javanainen, M., Metzler, R.: Anomalous diffusion of phospholipids and cholesterols in a lipid bilayer and its origins. Phys. Rev. Lett. 109, 188103 (2012)

34. Jespersen, S., Metzler, R., Fogedby, H.C.: Lévy flights in external force fields: Langevin and fractional Fokker-Planck equations and their solutions. Phys. Rev. E 59, 2736 (1999)

35. Kotera, K., Lemoine, M.: Inhomogeneous extragalactic magnetic fields and the second knee in the cosmic ray spectrum. Phys. Rev. D 77, 023005 (2008)

36. Kotulski, M.: Asymptotic distributions of continuous-time random walks: a probabilistic approach. J. Stat. Phys. 81, 777-792 (1995)

37. Landman, K.A., Pettet, G.J., Newgreen, D.F.: Mathematical models of cell colonization of uniformly growing domains. Bull. Math. Biol. 65, 235-262 (2003)

38. Le Vot, F., Abad, E., Yuste, S.B.: Continuous-time random-walk model for anomalous diffusion in expanding media. Phys. Rev. E 96, 032117 (2017)

39. Le Vot, F., Abad, E., Metzler, R., Yuste, S.B.: Continuous time random walk in a velocity field: role of domain growth, Galilei-invariant advection-diffusion, and kinetics of particle mixing. New J. Phys. 22, 073048 (2020)

40. Le Vot, F., Yuste, S.B., Abad, E.: Standard and fractional Ornstein-Uhlenbeck process on a growing domain. Phys. Rev. E 100, 012142 (2019)

41. Le Vot, F., Yuste, S.B.: Continuous-time random walks and Fokker-Planck equation in expanding media. Phys. Rev. E 98, 042117 (2018)

42. Metzler, R., Jeon, J.-H., Cherstvy, A.G., Barkai, E.: Anomalous diffusion models and their properties: non-stationarity, non-ergodicity, and ageing at the centenary of single particle tracking. Phys. Chem. Chem. Phys. 16, 24128-24164 (2014)

43. Metzler, R., Klafter, J.: The random walk's guide to anomalous diffusion: a fractional dynamics approach. Phys. Rep. 339, 1-77 (2000)

44. Montroll, E.W., Weiss, G.H.: Random walks on lattices. II. J. Math. Phys. 6, 167-181 (1965)

45. Montroll, E.. W.: Random walks on lattices. III. Calculation of first-passage times with application to exciton trapping on photosynthetic units. J. Math. Phys. 10, 753-765 (1969)

46. Metzler, R., Barkai, E., Klafter, J.: Anomalous diffusion and relaxation close to thermal equilibrium: a fractional Fokker-Planck equation approach. Phys. Rev. Lett. 82, 3563 (1999)

47. Murray, J.. D.: Mathematical Biology. Vol II: Spatial Models and Biomedical Applications. Springer, New York (2003)

48. Nelson, E.: Dynamical Theories of Brownian Motion. Princeton University Press, Princeton (1967)

49. Norregaard, K., Metzler, R., Ritter, C.M., Berg-Sørensen, K., Oddershede, L.B.: Manipulation and motion of organelles and single molecules in living cells. Chem. Rev. 117, 4342-4375 (2017)

50. Podlubny, I.: Fractional Differential Equations. Academic Press, San Diego (1999)

51. Palyulin, V.V., Chechkin, A.V., Metzler, R.: Lévy flights do not always optimize random blind search for sparse targets. Proc. Natl. Acad. Sci. USA 111, 2931-2936 (2014)

52. Peacock, J.A.: Cosmological Physics. Cambridga University Press, Cambridge, England (1999)

53. Richardson, L.F.: Atmospheric diffusion shown on a distance-neighbour graph. Proc. R. Soc. A 110, 709-737 (1926)

54. Reverey, J.F., Jeon, J.-H., Leippe, M., Metzler, R., Selhuber-Unkel, C.: Superdiffusion dominates intracellular particle motion in the supercrowded cytoplasm of pathogenic Acanthamoeba castellanii. Sci. Rep. 5, 11690 (2015)

55. Raichlen, D.A., Wood, B.M., Gordon, A.D., Mabulla, A.Z., Marlowe, F.W., Pontzer, H.: Evidence of Lévy walk foraging patterns in human hunter-gatherers. Proc. Natl. Acad. Sci. USA 111, 728-733 (2014)

56. Ryden, B.: Introducion to Cosmology. Addison-Wesley, San Francisco (2003)

57. Sancho, J.M., Lacasta, A.M., Lindenberg, K., Sokolov, I.M., Romero, A.H.: Diffusion on a solid surface: anomalous is normal. Phys. Rev. Lett. 92, 250601 (2004)

58. Scalas, E.: The application of continuous-time random walks in finance and economics. Phys. A 362, 225-239 (2006)

59. Scher, H., Montroll, E.W.: Anomalous transit-time dispersion in amorphous solids. Phys. Rev. B 12, 2455 (1975)

60. Szymanski, J., Weiss, M.: Elucidating the origin of anomalous diffusion in crowded fluids. Phys. Rev. Lett. 103, 038102 (2009)

61. Song, M.S., Moon, H.C., Jeonm, J.-H., Park, H.Y.: Neuronal messenger ribonucleoprotein transport follows an aging Lévy walk. Nat. Commun. 9, 344 (2018) 
62. Simpson, M.J., Sharp, J.A., Morrow, L.C., Baker, R.E.: Exact solutions of coupled multispecies linear reaction-diffusion equations on a uniformly growing domain. PLoS ONE 10, e0138894 (2015)

63. Simpson, M.J., Sharp, J.A., Baker, R.E.: Survival probability for a diffusive process on a growing domain. Phys. Rev. E 91, 042701 (2015)

64. Tabei, S.M., Burov, S., Kim, H.Y., Kuznetsov, A., Huynh, T., Jureller, J., Philipson, L.H., Dinner, A.R., Scherer, N.F.: Intracellular transport of insulin granules is a subordinated random walk. Proc. Natl. Acad. Sci. USA 110, 4911-4916 (2013)

65. Viswanathan, G.M., Afanasyev, V., Buldyrev, S.V., Murphy, E.J., Prince, P.A., Stanley, H.E.: Lévy flight search patterns of wandering albatrosses. Nature 381, 413-415 (1996)

66. Viswanathan, G.M., da Luz, M.G.E., Raposo, E.P., Stanley, H.E.: The Physics of Foraging. Cambridge University Press, Cambridge (2011)

67. Weigel, A.V., Simon, B., Tamkun, M.M., Krapf, D.: Ergodic and nonergodic processes coexist in the plasma membrane as observed by single-molecule tracking. Proc. Natl. Acad. Sci. USA 108, 6438-6443 (2011)

68. Weber, S.C., Spakowitz, A.J., Theriot, J.A.: Bacterial chromosomal loci move subdiffusively through a viscoelastic cytoplasm. Phys. Rev. Lett. 104, 238102 (2010)

69. Xu, P.B., Deng, W.H., Sandev, T.: Lévy walk with parameter dependent velocity: Hermite polynomial approach and numerical simulation. J. Phys. A 53, 115002 (2020)

70. Xu, P.B., Zhou, T., Metzler, R., Deng, W.H.: Lévy walk dynamics in an external harmonic potential. Phys. Rev. E 101, 062127 (2020)

71. Yates, C.A.: Discrete and continuous models for tissue growth and shrinkage. J. Theor. Biol. 350, 37-48 (2014)

72. Yuste, S.B., Abad, E., Escudero, C.: Diffusion in an expanding medium: Fokker-Planck equation, Green's function and first-passage properties. Phys. Rev. E 94, 032118 (2016)

73. Zaburdaev, V., Denisov, S., Klafter, J.: Lévy walks. Rev. Mod. Phys. 87, 483 (2015)

74. Zhou, T., Xu, P.B., Deng, W.H.: Lévy walk dynamics in mixed potentials from the perspective of random walk theory. Phys. Rev. E 103, 032151 (2021)

75. Zhou, T., Xu, P.B., Deng, W.H.: Lévy walk dynamics in non-static media. J. Phys. A 55, 025001 (2022)

Publisher's Note Springer Nature remains neutral with regard to jurisdictional claims in published maps and institutional affiliations. 